\title{
Accuracy of the adaptive GRP scheme and the simula- tion of 2-D Riemann problems for compressible Euler equations
}

\author{
Ee $\operatorname{Han}^{1}$, Jiequan $\mathrm{Li}^{2}$,* and Huazhong Tang ${ }^{3}$ \\ ${ }^{1}$ IAN, Department of Mathematics, Magdeburg University, D-31906, Germany \\ 2 School of Mathematical Science, Capital Normal University, Beijing 100048, P. R. \\ China \\ ${ }^{3}$ CAPT and LMAM, School of Mathematical Sciences, Peking University, Beijing 100871, \\ P. R. China
}

\begin{abstract}
The adaptive generalized Riemann problem (GRP) scheme for 2-D compressible fluid flows has been proposed in [25, Han, Li and Tang, An adaptive GRP scheme for compressible fluid flows, J. Comput. Phys., 229 (2010), 1448-1466] and it displays the capability in overcoming difficulties such as the start-up error for a single shock, and the numerical instability of the almost stationary shock. In this paper, we will provide the accuracy study and particularly show the performance in simulating $2-\mathrm{D}$ complex wave configurations formulated with the $2-\mathrm{D}$ Riemann problems for compressible Euler equations. For this purpose, we will first review the GRP scheme briefly when combined with the adaptive moving mesh technique and consider the accuracy of the adaptive GRP scheme via the comparison with the explicit formulae of analytic solutions of planar rarefaction waves, planar shock waves, the collapse problem of a wedge-shaped dam and the spiral formation problem. Then we simulate the full set of wave configurations in the 2-D four-wave Riemann problems for compressible Euler equations [60, Zhang and Zheng, Conjecture on the structure of solutions of the Riemann problem for 2-D gas dynamics systems, SIAM J. Math. Anal., 21 (1990), 593-630], including the interactions of strong shocks (shock reflections), vortex-vortex and shock-vortex etc. This study combines the theoretical results with the numerical simulations, and thus demonstrates what Ami Harten observed "for computational scientists there are two kinds of truth: the truth that you prove, and the truth you see when you compute" [33, Lax, Computational Fluid Dynamics, J. Sci. Comput., 31 (2007), 185-193].
\end{abstract}

AMS subject classifications: 65M06, 76M12, 35L60

Key words: Adaptive GRP scheme, 2-D Riemann problems, collapse of a wedge-shaped dam, spiral formation, shock reflections, vortex-shock interaction.

\section{Introduction}

The generalized Riemann problem (GRP) method was originally devised in [1] for one dimensional (1-D) system of an unsteady and inviscid flow by way of replacing the initial data with a piecewise linear function and analytically solving a generalized Riemann problem at each cell interface so as to yield numerical fluxes. Then it was extensively applied to simulating a large variety of wave configurations in 2-D and 3-D, including gas dynamics problems and combustion problems [3,7],

${ }^{*}$ Corresponding author. Email addresses: eehan84@yahoo.com (E. Han), jiequan@mail.cnu.edu.cn (J. Li), hztang@math.pku.edu.cn (H. Tang) 
nozzle flows with complex geometry [23] etc. The comprehensive description can be found in [4] and references therein. This pioneering derivation has two related versions, the Lagrangian and the Eulerian. The Eulerian version is always derived by using the Lagrangian version. The approach using the Lagrangian framework has the advantage that the contact discontinuity in each local wave pattern is always fixed with speed zero and the rarefaction waves and/or shock waves are located on either side. However, the passage from the Lagrangian version to the Eulerian is sometimes quite delicate, particularly for sonic cases and multi-dimensional applications. In order to efficiently deal with the sonic cases and apply to multi-dimensional systems, the second author and his coauthors introduced a direct Eulerian GRP scheme first for the shallow water equations [35] and for the Euler equations $[5,6]$, which used the main ingredient of Riemann invariants to decompose the strong coupling of nonlinear waves into a form of their simple superposition so that the rarefaction waves could be analytically resolved in a quite straightforward way. The numerical implementation is almost the same as that of the linearized Euler equations, and at each cell interface only a pair of linear algebraic equations are required to be solved. In [25] this direct Eulerian version was combined with the adaptive moving mesh method [51], which consisted of two independent parts: Evolution of PDEs with the GRP scheme on an quadrangular mesh and the mesh redistribution with the GaussSeidel iteration method. Such an adaptivity can overcome some drawbacks of many Godunov-type schemes, such as the instability of stationary shocks and start-up errors in a single shock wave simulation. Indeed, adaptive moving mesh methods have been successfully applied in a variety of scientific and engineering areas such as fluid dynamics and solid mechanics etc., where singular or nearly singular solutions are developed dynamically in fairly localized regions. To resolve the large solution variations requires extremely fine meshes over a small portion of the physical domain, see [8] for some practical examples. Successful implementation of an adaptive strategy can effectively decrease the computational cost and increase accuracy of the numerical approximations, see e.g. $[19,21,23,30,50,51,55,59]$. Up to now, there have been many important progresses in adaptive moving mesh methods for partial differential equations, including grid redistribution approach based on the variational principle of Winslow [56], Brackbill [9,10], Wang and Wang [55]; moving finite element methods of Miller and Miller [44], Davis and Flaherty [20]; moving mesh PDEs methods of Russell et al. [11,12]; and moving mesh methods based on the harmonic mapping of Dvinsky [22], and $\mathrm{Li}$ et al. [41,42].

In [25] we have validated the efficiency of the adaptive scheme in several aspects such as the CPU time, the start errors for single shock and the resolution of stationary shocks. However, the accuracy and numerical performance for complex wave configurations are awaiting for further investigation. Indeed, the convergence and stability of most numerical schemes are still huge challenges at present stage for (multidimensional) systems, even from the numerical viewpoint. It is of fundamental importance to provide a wide range of different exact solutions in order to numerically assess the accuracy etc. For this purpose, we provide the explicit formulae of the analytic solutions of several problems of 2-D compressible Euler equations from some recent theoretical studies [34, 38, 39]: Single oblique (shock and rarefaction) waves, the solution of the collapse problem of a wedgeshaped dam and axially symmetric spiral solutions. These explicit solutions are then used to study the accuracy of the adaptive GRP scheme. For the shock case, the accuracy is slightly of more than first order, but for continuous solutions (even rarefaction waves) the accuracy can attain more than one and half order.

Another aim of this paper is to check the capability of the adaptive GRP scheme to capture complex 2-D wave patterns. We choose the 2-D Riemann problems formulated in the pioneering work [60]. The 2-D Riemann problems reveal almost all substantial wave patterns of (regular, 
Mach) shock reflections, spiral formations (vortex-vortex interaction), vortex-shock interactions and so on, through simple classification of initial data. The rich wave configurations conjectured in [60] have been confirmed numerically by several subsequent works $[15,32,37,43,46,61]$. We simulate each wave configuration and demonstrate the excellent robustness of the adaptive GRP scheme. Compared with the existed numerical results obtained by other schemes, our results exhibit some excellences such as the resolution of small scale vortices and spirals. To our knowledge, it is necessary to require higher order accurate schemes generally in order to resolve such small scale phenomena. To some extent, the present study combines the theoretical results with the numerical simulations for a substantial set of 2-D wave configurations through Riemann-type problems. It demonstrates what Ami Harten observed "for computational scientists there are two kinds of truth: the truth that you prove, and the truth you see when you compute" [33].

Our paper is organized as follows. The adaptive GRP scheme is briefly reviewed in Section 2 and the accuracy of the scheme is demonstrated in Section 3 via the comparison with the explicit formulae of several interesting solutions. The complex wave configurations are carefully simulated in Section 4 via the 2-D four-wave Riemann problems formulated in [60]. Finally we place the review of the setting of 2-D Riemann problems including the explicit formulae of the analytic solutions in Appendix A.

\section{Brief Review of the adaptive GRP scheme}

This section briefly reviews the adaptive GRP scheme developed in [25] for the 2-D compressible Euler equations,

$$
\boldsymbol{U}=\left(\begin{array}{c}
\rho \\
\rho u \\
\rho v \\
E
\end{array}\right), \quad \boldsymbol{F}(\boldsymbol{U})=\left(\begin{array}{c}
\rho u \\
\rho u^{2}+p \\
\rho u v \\
u(E+p)
\end{array}\right), \boldsymbol{G}(\boldsymbol{U})=\left(\begin{array}{c}
\rho v \\
\rho u v \\
\rho v^{2}+p \\
v(E+p)
\end{array}\right),
$$

where $\rho, p, \boldsymbol{u}=(u, v)^{T}$ represent the density, the pressure and the velocity vector, the total energy is expressed as $E=\frac{1}{2} \rho\left(u^{2}+v^{2}\right)+\rho e$. This work will only focus on the polytropic gas with the state equation $e=\frac{p}{(\gamma-1) \rho}, \gamma>1$. The adaptive GRP scheme presented in [25] is based on the finite volume formulation of (2.1) over arbitrary quadrangular meshes.

\subsection{The GRP scheme on the quadrangular meshes}

Given a (physical) domain $\Omega_{p}$, we partition it as $\Omega_{p}=\bigcup_{(i, j) \in J_{\Omega}} A_{i, j}$, where $J_{\Omega}$ is an index set, and $A_{i, j}$ is an arbitrary quadrangle with four vertices $\boldsymbol{x}_{i+p, j+q}=\left(x_{i+p, j+q}, y_{i+p, j+q}\right), p, q= \pm \frac{1}{2}$. Let $\boldsymbol{x}=\boldsymbol{x}(\overline{\boldsymbol{\zeta}})$ the coordinate transformation from the computational domain $\Omega_{c}$ to the physical domain $\Omega_{p}$, and the cell $A_{i, j}$ is mapped into a rectangle, where $\boldsymbol{x}=(x, y)$ and $\overline{\boldsymbol{\zeta}}=(\bar{\xi}, \bar{\eta})$. We would like to emphasize that in the present paper the bold variables are used to denote vectors. For each the cell $A_{i, j}$ denote by $\boldsymbol{x}^{k}=\left(x^{k}, y^{k}\right)$ the four vertices of $A_{i, j}, k=1,2,3,4$, such that $\boldsymbol{x}^{1}=\left(x^{1}, y^{1}\right)=\left(x_{i-\frac{1}{2}, j-\frac{1}{2}}, y_{i-\frac{1}{2}, j-\frac{1}{2}}\right)$ 
and they are ordered in the counter-clockwise manner. Denote again by $C_{i, j}=A_{i, j} \times\left[t_{n}, t_{n+1}\right)$ a hexahedral control volume with four lateral faces $S_{k}$, and further by $A_{i, j}^{k}$ the $k^{\text {th }}$ neighboring quadrangle to $A_{i, j}$. The notation $\ell_{k}$ is the common boundary of $S_{k}, A_{i, j}^{k}$ and $A_{i, j}$; and $\boldsymbol{x}^{\tilde{k}}=\left(x^{\tilde{k}}, y^{\tilde{k}}\right)$ is the middle point of $\ell_{k}$, as shown in Figure 2.1.
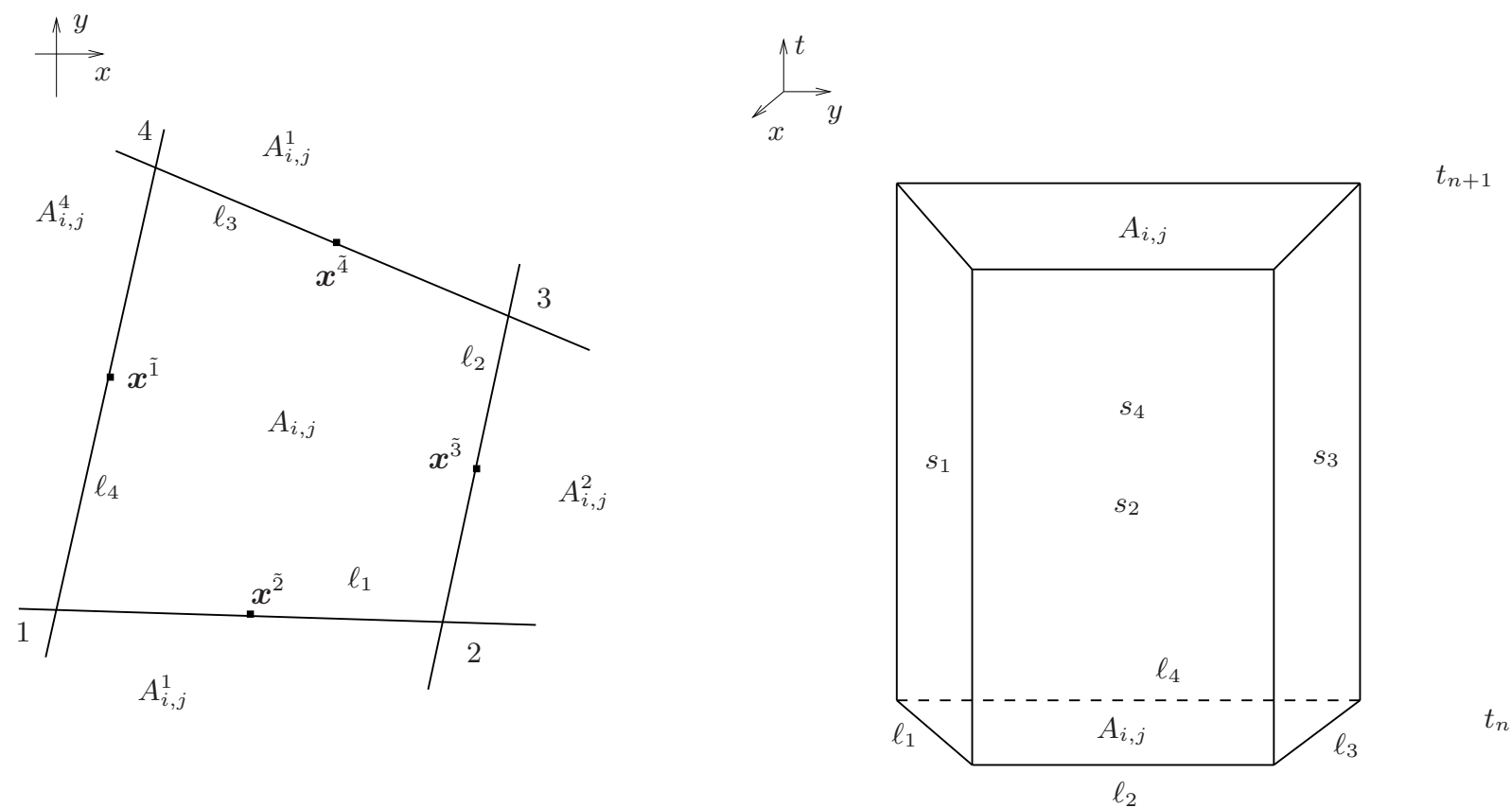

Figure 2.1: Quadrangle meshes and hexahedral control volumes.

We describe the GRP scheme on the fixed quadrangular meshes, starting from the finite volume formulation on $C_{i, j}$,

$$
\left|A_{i, j}\right| \boldsymbol{U}_{i, j}^{n+1}=\left|A_{i, j}\right| \boldsymbol{U}_{i, j}^{n}-\sum_{k=1}^{4} \int_{S_{k}}\left[\boldsymbol{F}(\boldsymbol{U}) \mu_{k}+\boldsymbol{G}(\boldsymbol{U}) \nu_{k}\right] d s,
$$

where $\left|A_{i, j}\right|$ is the area of $A_{i, j}, \boldsymbol{U}_{i, j}^{n}$ is the cell average of $\boldsymbol{U}\left(x, y, t_{n}\right)$ over $A_{i, j},\left(\mu_{k}, \nu_{k}\right)$ is the unit outer normal of $\ell_{k}$, pointing from $A_{i, j}$ to $A_{i, j}^{k}$. Then such a GRP scheme proceeds in the following three steps.

Step 1. Resolution of the generalized Riemann problem with the piecewise linear data. Denote by $\boldsymbol{x}_{i, j}$ the centroid of $A_{i, j}$. Given the piecewise linear data at the time $t=t_{n}$,

$$
\boldsymbol{U}_{A_{i, j}}\left(x, y, t_{n}\right)=\boldsymbol{U}_{i, j}^{n}+\left(x-x_{i, j}\right)\left(\boldsymbol{\sigma}_{x}\right)_{i, j}^{n}+\left(y-y_{i, j}\right)\left(\boldsymbol{\sigma}_{y}\right)_{i, j}^{n}, \quad(x, y) \in A_{i, j},
$$

we define the generalized Riemann problem for the planar Euler equations,

$$
\begin{aligned}
& \frac{\partial \boldsymbol{U}}{\partial t}+\frac{\partial \boldsymbol{H}\left(\boldsymbol{U} ; \mu_{k}, \nu_{k}\right)}{\partial \zeta}=0, \\
& \boldsymbol{U}(\zeta, 0)=\left\{\begin{array}{cc}
\boldsymbol{U}_{L, k}+\zeta \boldsymbol{U}_{L, k}^{\prime}, & \zeta<0, \\
\boldsymbol{U}_{R, k}+\zeta \boldsymbol{U}_{R, k}^{\prime}, & \zeta>0,
\end{array}\right.
\end{aligned}
$$


where $\zeta=\mu_{k} x+\nu_{k} y$, the directional flux functions $\boldsymbol{H}\left(\boldsymbol{U} ; \mu_{k}, \nu_{k}\right)=\boldsymbol{F}(\boldsymbol{U}) \mu_{k}+\boldsymbol{G}(\boldsymbol{U}) \nu_{k}, k=1,2,3,4$, and

$$
\left\{\begin{array} { c } 
{ \boldsymbol { U } _ { L , k } = \boldsymbol { U } _ { A _ { i , j } } ( x ^ { \tilde { k } } , y ^ { \tilde { k } } , t ^ { n } ) , } \\
{ \boldsymbol { U } _ { R , k } = \boldsymbol { U } _ { A _ { i , j } ^ { k } } ( x ^ { \tilde { k } } , y ^ { \tilde { k } } , t ^ { n } ) , }
\end{array} \quad \left\{\begin{array}{l}
\boldsymbol{U}_{L, k}^{\prime}=\left(\boldsymbol{\sigma}_{x}\right)_{i, j}^{n} \mu_{k}+\left(\boldsymbol{\sigma}_{y}\right)_{i, j}^{n} \nu_{k}, \\
\boldsymbol{U}_{R, k}^{\prime}=\left(\boldsymbol{\sigma}_{x}\right)_{A_{i, j}^{k}}^{n} \mu_{k}+\left(\boldsymbol{\sigma}_{y}\right)_{A_{i, j}^{k}}^{n} \nu_{k} .
\end{array}\right.\right.
$$

Note that the variable $\zeta$, different from $(\xi, \eta)$ introduced before, represents the normal coordinate component of the plane with normal $(\mu, \nu)$. Problem (2.5) is the so-called generalized planar Riemann problem. Once this generalized Riemann problem is solved so as to obtain the interface values $\boldsymbol{U}_{S_{k}}^{n}$ and $\left(\frac{\partial U}{\partial t}\right)_{S_{k}}^{n}$, we can define the "mid-point" value on the interface $S_{k}$ with the formula

$$
\boldsymbol{U}_{S_{k}}^{n+\frac{1}{2}}=\boldsymbol{U}_{S_{k}}^{n}+\frac{\Delta t}{2}\left(\frac{\partial \boldsymbol{U}}{\partial t}\right)_{S_{k}}^{n},
$$

used in numerical fluxes of the GRP scheme. The resolution of the generalized Riemann problem (2.4) can be referred to [6] or [25] for details of the calculation of $\boldsymbol{U}_{S_{k}}^{n}$ and $\left(\frac{\partial \boldsymbol{U}}{\partial t}\right)_{S_{k}}^{n}$.

Step 2. Evolution of the solutions. Define the numerical fluxes

$$
\boldsymbol{Q}_{k}=\left[\boldsymbol{F}\left(\boldsymbol{U}_{S_{k}}^{n+\frac{1}{2}}\right) \mu_{k}+\boldsymbol{G}\left(\boldsymbol{U}_{S_{k}}^{n+\frac{1}{2}}\right) \nu_{k}\right]\left|S_{k}\right|,
$$

where $\left|S_{k}\right|=\left|\ell_{k}\right| \Delta t$ is the area of $S_{k},\left|\ell_{k}\right|$ is the length of the edge $\ell_{k}, k=1,2,3,4$. Then (2.2) are approximated as

$$
\boldsymbol{U}_{i, j}^{n+1}=\boldsymbol{U}_{i, j}^{n}-\frac{1}{\left|A_{i, j}\right|} \sum_{k=1}^{4} \boldsymbol{Q}_{k}
$$

where the area $\left|A_{i, j}\right|=\frac{1}{2}\left[\left(x^{3}-x^{1}\right)\left(y^{4}-y^{2}\right)-\left(x^{4}-x^{2}\right)\left(y^{3}-y^{1}\right)\right]$.

Step 3. Slope updating. We update slopes $\left(\sigma_{z}\right)_{i, j}^{n+1}$ ( the subscript $z$ represents $x$ or $y$ ) in $(2.3$ ) by the following approximate procedure. Define

$$
\boldsymbol{U}_{S_{k}}^{n+1,-}:=\boldsymbol{U}_{S_{k}}^{n}+\Delta t\left(\frac{\partial \boldsymbol{U}}{\partial t}\right)_{S_{k}}^{n}, \quad k=1,2,3,4 .
$$

Calculate the slopes $\sigma_{\bar{\xi}}$ and $\sigma_{\bar{\eta}}$ as

$$
\sigma_{q}^{n+1,-}:= \begin{cases}\frac{1}{\Delta \bar{\eta}}\left(U_{S_{3}}^{n+1,-}-U_{S_{1}}^{n+1,-}\right), & q=\bar{\eta}, \\ \frac{1}{\Delta \bar{\xi}}\left(U_{S_{2}}^{n+1,-}-U_{S_{4}}^{n+1,-}\right), & q=\bar{\xi},\end{cases}
$$

and

$$
\sigma_{q}^{n+1}= \begin{cases}\operatorname{minmod}\left(\beta \frac{U_{i, j+1}^{n+1}-U_{i, j}^{n+1}}{\Delta \bar{\eta}}, \sigma_{q}^{n+1,-}, \beta \frac{U_{i, j}^{n+1}-U_{i, j-1}^{n+1}}{\Delta \bar{\eta}}\right), & q=\bar{\eta}, \\ \operatorname{minmod}\left(\beta \frac{U_{i+1, j}^{n+1}-U_{i, j}^{n+1}}{\Delta \bar{\xi}}, \sigma_{q}^{n+1,-}, \beta \frac{U_{i, j}^{n+1}-U_{i-1, j}^{n+1}}{\Delta \bar{\xi}}\right), & q=\bar{\xi},\end{cases}
$$


where $\Delta \bar{\xi}$ and $\Delta \bar{\eta}$ are the side length of the rectangular meshes covering the computational domain $\Omega_{c}$ and $\beta \in[0,2)$. Then $\left(\boldsymbol{\sigma}_{z}\right)_{i, j}^{n+1}$ (the subscript $z=x$ or $y$ ) are obtained by

$$
\boldsymbol{\sigma}_{x}=\frac{1}{J}\left[\boldsymbol{\sigma}_{\bar{\xi}} y_{\bar{\eta}}-\boldsymbol{\sigma}_{\bar{\eta}} y_{\bar{\xi}}\right], \quad \boldsymbol{\sigma}_{y}=\frac{1}{J}\left[-\boldsymbol{\sigma}_{\bar{\xi}} x_{\bar{\eta}}+\boldsymbol{\sigma}_{\bar{\eta}} x_{\bar{\xi}}\right]
$$

where the Jacobian $J=x_{\bar{\xi}} y_{\bar{\eta}}-x_{\bar{\eta}} y_{\bar{\xi}}$ and

$$
\begin{array}{ll}
x_{\bar{\xi}}=x^{\overline{2}}-x^{\overline{4}}, & x_{\bar{\eta}}=x^{\overline{3}}-x^{\overline{1}}, \\
y_{\bar{\xi}}=y^{\overline{2}}-y^{\overline{4}}, & y_{\bar{\eta}}=y^{\overline{3}}-y^{\overline{1}} .
\end{array}
$$

The above indices are referred to Figure 2.1.

\subsection{Adaptive mesh redistribution}

This subsection illustrates the adaptive mesh redistribution based on the variational formulation briefly.

\subsubsection{Mesh redistribution}

Let $\boldsymbol{x}=\boldsymbol{x}(\overline{\boldsymbol{\zeta}})$ be the coordinate map from the computational domain $\Omega_{c}=[0,1] \times[0,1]$ to the physical domain $\Omega_{p}=[a, b] \times[c, d]$, and $\overline{\boldsymbol{\zeta}}=\overline{\boldsymbol{\zeta}}(\boldsymbol{x})$ denote its inversion, where $\boldsymbol{x}=(x, y)$ and $\overline{\boldsymbol{\zeta}}=(\bar{\xi}, \bar{\eta})$. The map $\boldsymbol{x}=\boldsymbol{x}(\overline{\boldsymbol{\zeta}})$ are regarded as the solution of a "mesh-energy" functional,

$$
E(\boldsymbol{x})=\frac{1}{2} \int_{\Omega_{c}}\left[\bar{\nabla}^{\top} x G \bar{\nabla} x+\bar{\nabla}^{\top} y G \bar{\nabla} y\right] d \bar{\xi} d \bar{\eta}
$$

where $\bar{\nabla}=\left(\partial_{\bar{\xi}}, \partial_{\bar{\eta}}\right)^{\top}, G$ is a given symmetric positive definite matrix depending on the underlying solution to be adapted. In particular, we often use $G=\omega I$, where $I$ is the identity matrix, and the monitor function $\omega$ is a positive weighted function. This produces an isotropic mesh adaptation.

The corresponding Euler-Lagrange equations of (2.13) for $G=\omega I$ are

$$
\left\{\begin{array}{c}
\left(\omega x_{\bar{\xi}}\right)_{\bar{\xi}}+\left(\omega x_{\bar{\eta}}\right)_{\bar{\eta}}=0 \\
\left(\omega y_{\bar{\xi}}\right)_{\bar{\xi}}+\left(\omega y_{\bar{\eta}}\right)_{\bar{\eta}}=0
\end{array}\right.
$$

This system will be solved with the boundary conditions $x(0, \bar{\eta})=a, x(1, \bar{\eta})=b, y(\bar{\xi}, 0)=c$, and $y(\bar{\xi}, 1)=d$. 


\subsubsection{Mesh adaptation}

The Gauss-Seidel iteration method are used to solve the mesh-moving equations in (2.14):

$$
\begin{gathered}
\alpha_{i+1, j+\frac{1}{2}}\left(x_{i+\frac{3}{2}, j+\frac{1}{2}}^{[\nu]}-x_{i+\frac{1}{2}, j+\frac{1}{2}}^{[\nu+1]}\right)-\alpha_{i, j+\frac{1}{2}}\left(x_{i+\frac{1}{2}, j+\frac{1}{2}}^{[\nu+1]}-x_{i-\frac{1}{2}, j+\frac{1}{2}}^{[\nu+1]}\right) \\
+\quad \beta_{i+\frac{1}{2}, j+1}\left(x_{i+\frac{1}{2}, j+\frac{3}{2}}^{[\nu]}-x_{i+\frac{1}{2}, j+\frac{1}{2}}^{[\nu+1]}\right)-\beta_{i+\frac{1}{2}, j}\left(x_{i+\frac{1}{2}, j+\frac{1}{2}}^{[\nu+1]}-x_{i+\frac{1}{2}, j-\frac{1}{2}}^{[\nu+1]}\right)=0, \\
\alpha_{i+1, j+\frac{1}{2}}\left(y_{i+\frac{3}{2}, j+\frac{1}{2}}^{[\nu]}-y_{i+\frac{1}{2}, j+\frac{1}{2}}^{[\nu+1]}\right)-\alpha_{i, j+\frac{1}{2}}\left(y_{i+\frac{1}{2}, j+\frac{1}{2}}^{[\nu+1]}-y_{i-\frac{1}{2}, j+\frac{1}{2}}^{[\nu+1]}\right) \\
+\quad \beta_{i+\frac{1}{2}, j+1}\left(y_{i+\frac{1}{2}, j+\frac{3}{2}}^{[\nu]}-y_{i+\frac{1}{2}, j+\frac{1}{2}}^{[\nu+1]}\right)-\beta_{i+\frac{1}{2}, j}\left(y_{i+\frac{1}{2}, j+\frac{1}{2}}^{[\nu+1]}-y_{i+\frac{1}{2}, j-\frac{1}{2}}^{[\nu+1]}\right)=0,
\end{gathered}
$$

where the coefficients are given by

$$
\alpha_{i, j+\frac{1}{2}}=\frac{1}{2}\left[\omega\left(U_{i, j}^{[\nu]}\right)+\omega\left(U_{i, j+1}^{[\nu]}\right)\right], \quad \beta_{i+\frac{1}{2}, j}=\frac{1}{2}\left[\omega\left(U_{i, j}^{[\nu]}\right)+\omega\left(U_{i+1, j}^{[\nu]}\right)\right] .
$$

\subsubsection{Conservative interpolation of the solutions}

After each iterative step of (2.14) (resp. (2.15)), we need to remap the approximate solutions from the old mesh $A_{i, j}:=A_{i, j}^{[\nu]}$ onto the newly resulting mesh $\widetilde{A}_{i, j}:=A_{i, j}^{[\nu+1]}$. Let $\widetilde{\boldsymbol{U}}_{i, j}:=\boldsymbol{U}_{i, j}^{[\nu+1]}$ and $\boldsymbol{U}_{i, j}:=\boldsymbol{U}_{i, j}^{[\nu]}$ be the new and old cell averages of the conservative vector $U$ over the cells $\widetilde{A}_{i, j}$ and $A_{i, j}$, respectively. The conservation interpolation used in [25] is

$$
\left|\tilde{A}_{i, j}\right| \widetilde{\boldsymbol{U}}_{i, j}=\left|A_{i, j}\right| \boldsymbol{U}_{i, j}-\left[\left(\boldsymbol{U} n_{c}\right)_{\ell_{4}}+\left(\boldsymbol{U} n_{c}\right)_{\ell_{2}}\right]-\left[\left(\boldsymbol{U} n_{c}\right)_{\ell_{3}}+\left(\boldsymbol{U} n_{c}\right)_{\ell_{1}}\right],
$$

where $n_{c}=c^{x} \mu+c^{y} \nu,\left(c^{x}, c^{y}\right)=(x-\tilde{x}, y-\tilde{y})$, and $\left(n_{c} U\right)_{\ell_{k}}$ denotes the value of the $n_{c} \boldsymbol{U}$ through the boundary $\ell_{k}$. In practice, we always use the following upwind approximation to define $\left(\boldsymbol{U} n_{c}\right)_{\ell_{k}}$ :

$$
\left(n_{c} \boldsymbol{U}\right)_{l_{k}}=\frac{\left(n_{c}\right)_{\ell_{k}}+\left|\left(n_{c}\right)_{\ell_{k}}\right|}{2}\left(\boldsymbol{U}_{L, k}\right)+\frac{\left(n_{c}\right)_{\ell_{k}}-\left|\left(n_{c}\right)_{\ell_{k}}\right|}{2}\left(\boldsymbol{U}_{R, k}\right),
$$

where $\boldsymbol{U}_{m, k}, m=L$ or $R$, is defined similar to $(2.5)$.

Besides, the adaptive GRP scheme needs to remap the approximate slopes of the solutions from the old meshes $\left\{A_{i, j}\right\}$ onto the new meshes $\left\{\widetilde{A}_{i, j}\right\}$. Here we use the conservative interpolation (2.16) by replacing $\boldsymbol{U}$ with $\boldsymbol{\sigma}_{z}$, where the subscript $z=x$ or $y$.

\subsubsection{Monitor function}

The monitor function is devised to detect the variation of physical solutions. One of the traditional choices is an arclength-type monitor (AL-monitor), such as

$$
\omega=\sqrt{1+\alpha|\nabla W|^{2}}
$$

or

$$
\omega=\sqrt{1+\alpha|\bar{\nabla} W|^{2}}
$$

where $\nabla=\left(\partial_{x}, \partial_{y}\right), \alpha$ is a nonnegative constant, and $W$ may represent certain physical variables, such as the density, the velocity, the internal energy and so on. The adaptive moving mesh method with these AL-monitors would make the meshes concentrate on around the large variation region so strictly that a discontinuity may be distorted easily. Generally, the monitor function produces the 
very singular meshes around the stiff solution areas. So commonly, we use some spatial smoothing procedure for the monitor function in order to avoid these drawbacks. Here we choose

$$
\begin{aligned}
\omega_{i, j} \longleftarrow & \frac{1}{4} \omega_{i, j}+\frac{1}{8}\left(\omega_{i, j+1}+\omega_{i, j-1}+\omega_{i+1, j}+\omega_{i-1, j}\right) \\
& +\frac{1}{16}\left(\omega_{i+1, j+1}+\omega_{i-1, j-1}+\omega_{i+1, j-1}+\omega_{i-1, j+1}\right) .
\end{aligned}
$$

\subsubsection{Outline of the adaptive GRP scheme}

The implementation of the adaptive GRP scheme is similar to the one set by Tang and Tang [50] except the remapping of the slopes. It is formulated with two independent steps: the mesh equations (2.14) are first solved by the Gauss-Seidel iteration (2.15) and the solutions and the approximate slopes are simultaneously remapped from the old meshes onto the new meshes; and then the compressible Euler equations are evolved by the GRP scheme on the fixed nonuniform meshes. We repeat these two steps until it reaches the output time $T$. The details of our algorithm are presented in the following.

Step 1. If $t_{n}=0$, give an initial (uniform or nonuniform) partition $\left\{A_{i, j}\right\}$ of the physical domain $\Omega_{p}$ and a uniform partition of the computational domain $\Omega_{c}$, and compute the cell average values $\boldsymbol{U}_{i, j}^{0}$ and the slopes $\left(\boldsymbol{\sigma}_{x}\right)_{i, j}^{0}$ and $\left(\boldsymbol{\sigma}_{y}\right)_{i, j}^{0}$.

If $t_{n}>0$, set $\boldsymbol{x}_{i, j}^{[0]}=\boldsymbol{x}_{i, j}^{n}, \boldsymbol{U}_{i, j}^{[0]}=\boldsymbol{U}_{i, j}^{n}$, and $\left(\boldsymbol{\sigma}_{z}^{[0]}\right)_{i, j}=\left(\boldsymbol{\sigma}_{z}\right)_{i, j}^{n}, z=x$ or $y$.

Step 2. For $\nu=0,1,2, \cdots, \mu-1$, redistribute the mesh as follows:

(a) Relocate the mesh points by the Gauss-Seidel iteration (2.15).

(b) Remap the solution vector $\left\{\boldsymbol{U}_{i, j}\right\}$ and the slopes $\left(\boldsymbol{\sigma}_{z}\right)_{i, j}(z=x$ or $y)$ from the old meshes $\left\{A_{i, j}^{[\nu]}\right\}$ onto the new meshes $\left\{A_{i, j}^{[\nu+1]}\right\}$ by using the conservation interpolation (2.16).

(c) Repeat steps (a) and (b) for a fixed number $\mu$ or until $\left.\sum_{i, j} \mid(x, y)_{i, j}^{[\nu+1]}-(x, y)_{i, j}^{[\nu]}\right) \mid \leq \varepsilon$.

Step 3. Set $A_{i, j}=A_{i, j}^{[\nu+1]}, \boldsymbol{U}_{i, j}^{n}=\boldsymbol{U}_{i, j}^{[\nu+1]},\left(\boldsymbol{\sigma}_{z}\right)_{i, j}^{n}=\left(\boldsymbol{\sigma}_{z}\right)_{i, j}^{[\nu+1]}(z=x$ or $y)$. Compute $\boldsymbol{U}_{i, j}^{n+1}$ and $\left(\sigma_{z}\right)_{i, j}^{n+1}(z=x$ or $y)$ by the GRP approach in Section 2.1 on the fixed mesh $A_{i, j}$.

Step 4. Go to Step 1 if $t_{n+1}<T$.

\section{Accuracy of adaptive GRP scheme via explicit solutions}

In [25] we have validated the current adaptive GRP scheme in terms of the CPU time, the start-up error for a single shock, and the numerical instability of the almost stationary shock etc. This section studies the accuracy of this scheme through the comparison of the single oblique wave, the collapse of wedge-shape dam and the axially symmetric solutions with the corresponding explicit solutions of the 2-D compressible Euler equations. They are adopted from some recent theoretical studies [34, 38,39] and provided in Appendix A. As is well-known, the problems with explicit solutions serve as benchmarks to testify numerical schemes. In the following, the Courant number is set to be 0.5 , the parameter $\beta=1.5$ in the slope updating and $\gamma=2.0$ unless explicitly stated.

\subsection{Propagation of a single oblique wave}

The adaptive GRP scheme is employed to simulate the propagation of a single oblique planar rarefaction wave and a single oblique shock wave. In Table 3.1, we show the numerical errors and 
Table 3.1: The $L^{2}$ error and the accuracy order for the oblique rarefaction wave case.

\begin{tabular}{|c|c|c|c|c|c|}
\hline $\mathrm{M}$ & 20 & 40 & 80 & 160 & 320 \\
\hline $\mathrm{GRP}$ & $1.09 e-4(-)$ & $3.90 e-5(1.50)$ & $1.06 e-5(1.88)$ & $2.89 e-6(1.83)$ & $8.43 e-7(1.82)$ \\
\hline $\mathrm{AGRP}$ & $4.37 e-5(-)$ & $1.74 e-5(1.33)$ & $5.96 e-6(1.55)$ & $1.59 e-6(1.91)$ & $3.69 e-7(2.10)$ \\
\hline
\end{tabular}

the rate of convergence of the GRP and the adaptive GRP schemes for the single oblique rarefaction wave case. The initial data are

$$
(\rho, u, v, p)(x, y, t=0)= \begin{cases}(1,0,0,1), & \text { for } \mu x+\nu y<0 \\ (0.5,-0.7785,0.28334,0.25), & \text { for } \mu x+\nu y>0\end{cases}
$$

where $(\mu, \nu)=\left(\sin \frac{7 \pi}{18},-\cos \frac{7 \pi}{18}\right)$. As the polytropic index $\gamma=2.0$, the Euler equations $(2.1)$ correspond to the shallow water equations.

In Table 3.2, we present a numerical comparison for the single oblique shock case, for which the initial data are

$$
(\rho, u, v, p)(x, y, t=0)= \begin{cases}(1.4,0,0,1), & \text { for } \mu x+\nu y<0 \\ (8,7.1447,-4.1251,116.5), & \text { for } \mu x+\nu y>0\end{cases}
$$

where $(\mu, \nu)=\left(\sin \frac{\pi}{3},-\cos \frac{\pi}{3}\right)$ and $\gamma=1.4$.

Table 3.2: The $L^{2}$ error and the accuracy order for the oblique shock case.

\begin{tabular}{|c|c|c|c|c|c|}
\hline $\mathrm{M}$ & 20 & 40 & 80 & 160 & 320 \\
\hline $\mathrm{GRP}$ & $1.82 e-1(-)$ & $9.96 e-2(0.87)$ & $5.53 e-2(0.85)$ & $2.92 e-2(0.92)$ & $1.52 e-2(0.94)$ \\
\hline $\mathrm{AGRP}$ & $1.20 e-1(-)$ & $6.07 e-2(0.98)$ & $2.69 e-2(1.17)$ & $1.30 e-2(1.05)$ & $6.35 e-3(1.10)$ \\
\hline
\end{tabular}

From Tables 3.1 and 3.2, we observe that for the rarefaction case (continuous solution), the accuracy can be of second order, while for the shock case (discontinuous solution), the accuracy is just of first order. However, the accuracy of the adaptive GRP scheme is obviously higher than the GRP scheme.

\subsection{Collapse of a wedge-shaped dam}

This problem boils down to the interaction of two planar rarefaction waves. Its precise set-up and the explicit formulae of the solution are referred to Appendix A. The initial density here is set to 
be $\rho_{1}=\left(\frac{1}{\gamma}\right)^{\frac{1}{(\gamma-1)}}$, but the propagation speed of the vacuum interface $(\bar{u}, \bar{v})$ is not specified here and it can be determined from the state $\left(\rho_{1}, 0,0\right)$. In Table 3.3 , we give the $L^{2}$ error and the convergence rate. The exact solution and the numerical solution by the adaptive GRP scheme are displayed in Figure 3.1.

Table 3.3: The $L^{2}$ error and the accuracy order for the dam collapse problem

\begin{tabular}{|c|c|c|c|c|c|}
\hline $\mathrm{M}$ & 20 & 40 & 80 & 160 & 320 \\
\hline $\mathrm{GRP}$ & $7.05 e-4(-)$ & $3.67 e-4(0.94)$ & $1.67 e-4(1.19)$ & $7.61 e-5(1.08)$ & $4.08 e-5(0.9)$ \\
\hline AGRP & $4.32 e-4(-)$ & $1.03 e-4(2.07)$ & $2.27 e-5(2.18)$ & $7.99 e-6(1.71)$ & $2.74 e-6(1.55)$ \\
\hline
\end{tabular}
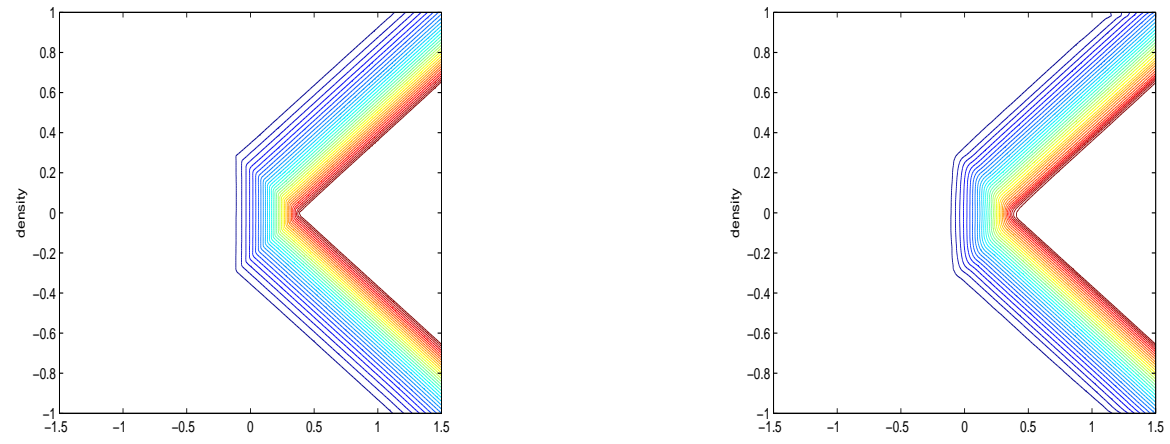

Figure 3.1: The dam collapse problem. The left is the exact solution and the right is the numerical solution with $160 \times 160$ adaptive meshes at $t=0.2$ and the monitor function $\omega=\sqrt{1+10|\bar{\nabla} \rho|^{2}}$.

We see that these numerical results match well the analytical counterpart. The accuracy order of the adaptive GRP scheme can attain the order of more than one and half, but the GRP scheme is only of first order. Since the solutions are just Lipschitz continuous, the GRP or the adaptive GRP scheme loses a little bit accuracy. This is not strange. Even for one-dimensional problems, numerical schemes lose their accuracy when resolving rarefaction waves.

\subsection{Axially symmetric problem}

We simulate the formation of spirals through this example. The initial data are

$$
(\rho, u, v)(x, y, 0)=(0.5, \sin \theta,-\cos \theta), \quad \theta \in[0,2 \pi) .
$$

Table 3.4 shows the $L^{2}$ error and the accuracy order of the standard GRP scheme and the adaptive GRP scheme. Figures 3.2-3.3 display the numerical solution by the adaptive GRP scheme and the exact solution.

Since the solution of this is more regular than the last example, the numerical accuracy is better. 
Table 3.4: The $L^{2}$ error and the accuracy order for axially symmetric problem

\begin{tabular}{|c|c|c|c|c|c|}
\hline $\mathrm{M}$ & 20 & 40 & 80 & 160 & 320 \\
\hline $\mathrm{GRP}$ & $4.16 e-4(-)$ & $1.02 e-4(1.92)$ & $3.41 e-5(1.70)$ & $1.15 e-5(1.57)$ & $4.60 e-6(1.32)$ \\
\hline $\mathrm{AGRP}$ & $2.52 e-4(-)$ & $6.78 e-5(1.82)$ & $1.83 e-5(1.90)$ & $5.44 e-6(1.72)$ & $1.50 e-6(1.89)$ \\
\hline
\end{tabular}

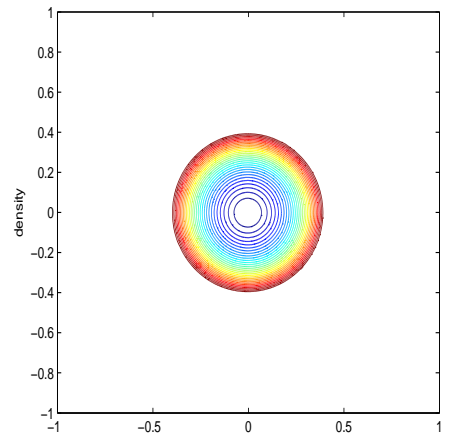

(a) Contour lines for density

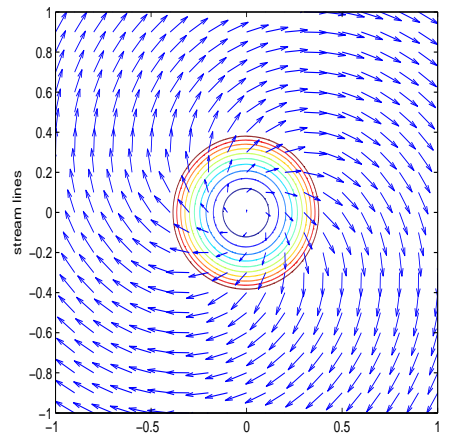

(b) Velocity field

Figure 3.2: Exact solution at $t=0.2$.

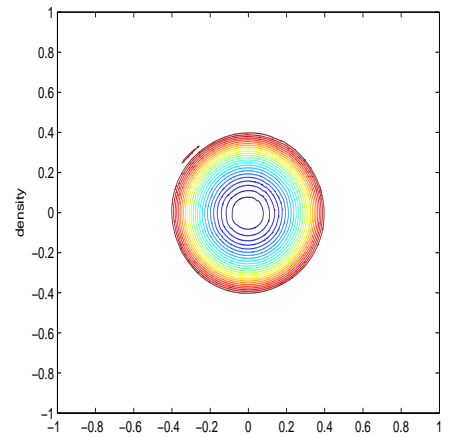

(a) Contour lines for density

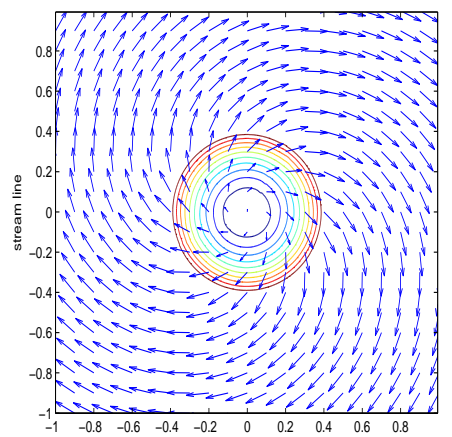

(b) Velocity field

Figure 3.3: Numerical solutions by the adaptive GRP scheme at time $t=0.2$ with the monitor function $\omega=$ $\sqrt{1+100\left(|\bar{\nabla} \rho|^{2}+|\bar{\nabla} u|^{2}+|\bar{\nabla} v|^{2}\right)}$ and $320 \times 320$ cells.

\section{The full set of 2-D Riemann problems and the simulations}

This section verifies the capability of the current adaptive GRP scheme in capturing complex 2-D wave configurations by simulating the $2-\mathrm{D}$ Riemann problems of the compressible Euler equations. The computational domain is $[-0.5,0.5] \times[-0.5,0.5]$, and the initial data comprise four different 
constant states,

$$
(\rho, u, v, p)(x, y, 0)=\left\{\begin{array}{lc}
\left(\rho_{1}, u_{1}, v_{1}, p_{1}\right), & 0<x<0.5,0<y<0.5, \\
\left(\rho_{2}, u_{2}, v_{2}, p_{2}\right), & -0.5<x<0,0<y<0.5, \\
\left(\rho_{3}, u_{3}, v_{3}, p_{3}\right), & -0.5<x<0,-0.5<y<0, \\
\left(\rho_{4}, u_{4}, v_{4}, p_{4}\right), & 0<x<0.5,-0.5<y<0 .
\end{array}\right.
$$

See Figure 4.1. As we mentioned in Appendix A.4, there are 19 different classifications of the 2-D Riemann problems under an appropriate restriction. Several numerical simulations $[32,37,43,46]$ have been done to verify those wave configurations conjectured in [60], including almost all essential 2-D physical phenomena such as shock reflections, the spiral formation (rolling up of slip lines), vortex-shock interactions etc. Nevertheless, many mysteries are awaiting for understanding and discovery. We will take the adaptive GRP method to simulate each configuration carefully and find small scale phenomena such as vortices in the interaction of vortex sheets with different signs. The parameter $\beta$ in the minmod limier is 1.5, the polytropic index $\gamma$ and the CFL number are taken as 1.4 and 0.5, respectively. All figures in this section are displayed with the density contour. We follow [37] to group all simulations into six categories: (i) the interaction of pure shocks; (ii) the interaction of pure vortex sheets; (iii) the interactions of vortex-shock; (iv) the interaction of pure rarefaction waves; (v) the interaction of vortex-rarefaction waves; and (vi) other configurations. The detailed classifications are listed in Appendix A.4.

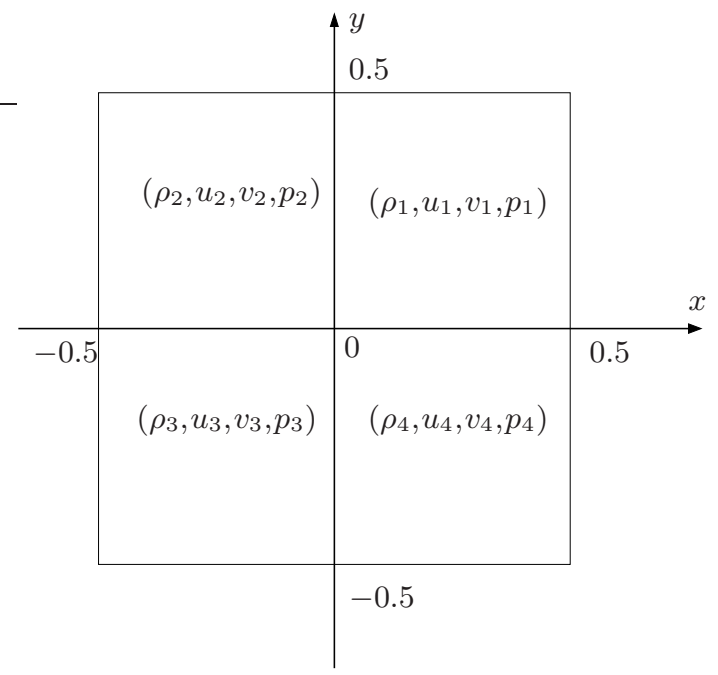

Figure 4.1: The initial data for the 2D Riemann problem.

\subsection{Interaction of pure planar shock waves}

The first group we want to simulate is the interaction of pure shocks, see [37]. There are two classes: The first is $S_{12}^{+} S_{23}^{+} S_{34}^{-} S_{41}^{-}$and the density contour is shown in Figure 4.2; the second is $S_{12}^{+} S_{23}^{-} S_{34}^{+} S_{41}^{-}$and the numerical results are displayed in Figures 4.3-4.5. Due to the choice of initial data, $S_{12}^{+}$and $S_{41}^{-}$(resp. $S_{23}^{+}$and $S_{34}^{-}, S_{23}^{-}$and $S_{34}^{+}$) have the same strength and moving 
speed, and thus they are symmetric with respect to $\xi-\eta=u_{1}-v_{1}$, which can be considered to be a rigid wall where $(\xi, \eta)=(x / t, y / t)$ are scaled spatial coordinates. We see the rich pictures of regular and Mach reflections of shocks that are disclosed in [8].

Let us first discuss the case depicted in Figure 4.2. Due to the symmetry, we just look at the upper part over the symmetrical line $\xi=\eta$. The shock $S_{23}^{-}$bifurcates at the trip point into a reflected shock, a Mach stem and a slip line. The reflected shock matches (interacts with) the shock $S_{12}^{+}$ to produce a new shock. The resulting pattern is extremely complicated. Around the trip point, there exist a lot of theoretical analyses to demonstrate the stability of the local structure, see [17] and references therein.

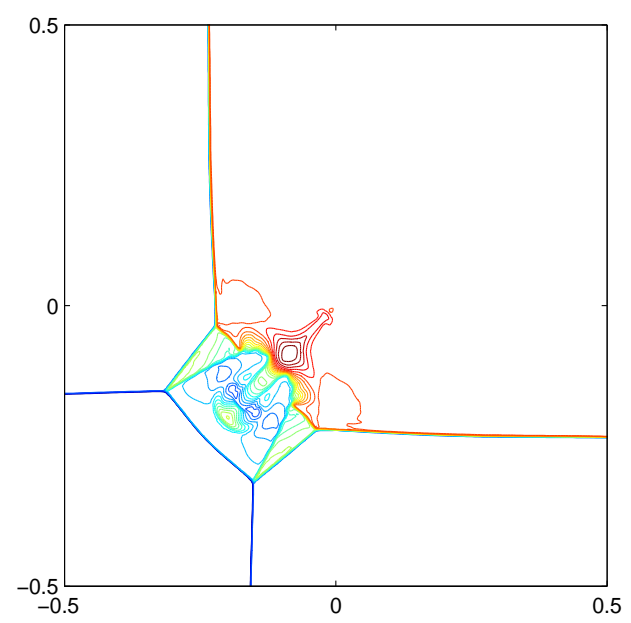

Figure 4.2: $\left[S_{12}^{+} S_{23}^{+} S_{34}^{-} S_{41}^{-}\right]$. The initial data are $\rho_{1}=1.5$, $u_{1}=0, v_{1}=0, p_{1}=1.5 ; \rho_{2}=0.5323, u_{2}=1.206, v_{2}=0, p_{2}=0.3$; $\rho_{3}=0.138, u_{3}=1.206, v_{3}=1.206, p_{3}=0.029 ; \rho_{4}=0.5323, u_{4}=$ $0, v_{4}=1.206, p_{4}=0.3$. The meshes are $200 \times 200$. The output time is $t=0.35$. The monitor function is $\omega=\sqrt{1+0.01|\bar{\nabla} e|^{2}}$.

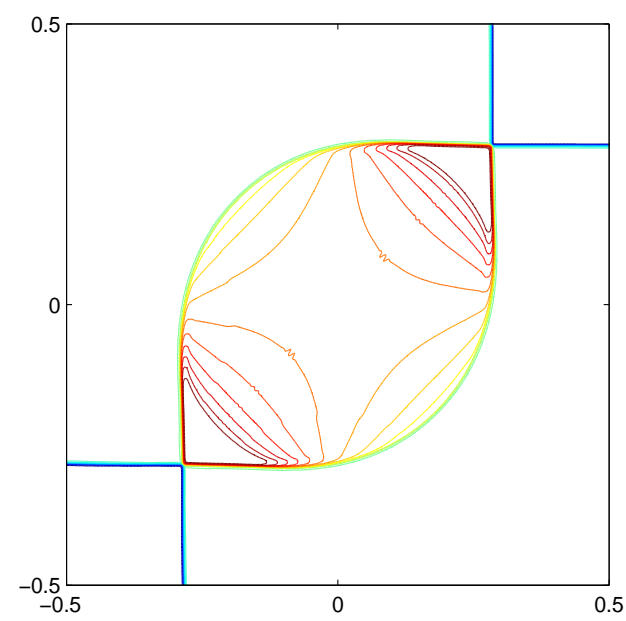

Figure 4.3: $\left[S_{12}^{+} S_{23}^{-} S_{34}^{+} S_{41}^{-}\right]$. The initial data are $\rho_{1}=1, u_{1}=$ $-0.189970, v_{1}=-0.189970, p_{1}=1.4 ; \rho_{2}=1.3, u_{2}=0.189970$, $v_{2}=-0.189970, p_{2}=2.025532 ; \rho_{3}=1, u_{3}=0.189970, v_{3}=$ $0.189970, p_{3}=1.4 ; \rho_{4}=1.3, u_{4}=-0.189970, v_{4}=0.189970$, $p_{4}=2.025532$. The output time is $t=0.2$. The meshes are $200 \times 200$. The monitor function is $\omega=\sqrt{1+5|\bar{\nabla} e|^{2}}$.

The second class $S_{12}^{+} S_{23}^{-} S_{34}^{+} S_{41}^{-}$has two symmetrical axes $\xi-\eta=u_{1}-v_{1}$ and $\xi+\eta=u_{2}+v_{2}$ and shows us a complete series of pictures from the regular reflection of shocks to the double Mach reflection, depending on the distribution of initial data. Figure 4.3 is for the standard regular shock reflection: shock $S_{23}^{+}$collides the line $\xi-\eta=u_{1}-v_{1}$ (rigid wall) and is reflected. Similarly for other three shocks. The reflected shocks match together to form a global pattern. Figures 4.4 and 4.5 show a simple Mach reflection of shocks and a double Mach reflection, respectively. From these examples, we see that our adaptive GRP scheme has sharp capability of capturing 2-D shocks with relatively few mesh points.

We recall that many theoretical attempts have been made to understand the transition criterion from the regular reflection to the simple or even the double Mach reflection, but it is very difficult. The earliest contribution seems due to [40] and later on it was refined in [13], [47] and references therein. Comprehensive descriptions can be found in [8]. Recently, some progresses have been made for the regular reflection by using the potential flow equation [16] and the stability of the Mach reflection structure [17] by using the full Euler equations. 


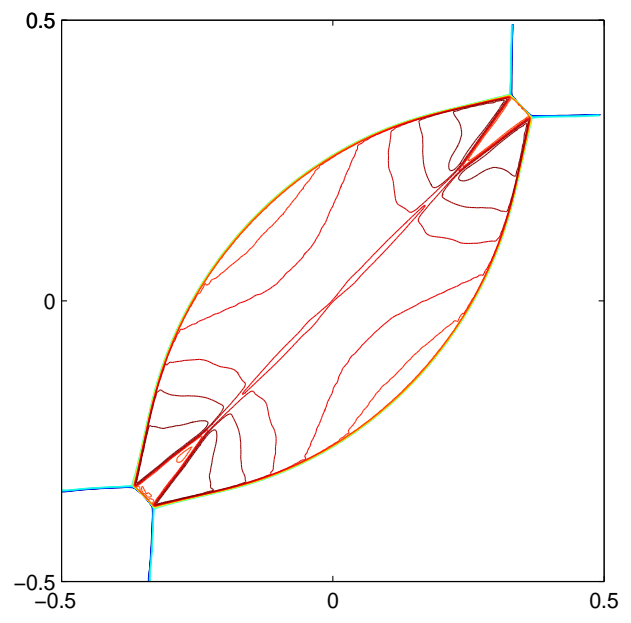

Figure 4.4: $\left[S_{12}^{+} S_{23}^{-} S_{34}^{+} S_{41}^{-}\right]$. The initial data are $\rho_{1}=1, u_{1}=$ $-0.5612, v_{1}=-0.5612, p_{1}=0.7 ; \rho_{2}=2.5, u_{2}=0.5612, v_{2}=$ $-0.5612, p_{2}=2.8 ; \rho_{3}=1, u_{3}=0.5612, v_{3}=0.5612, p_{3}=0.7$; $\rho_{4}=2.5, u_{4}=-0.5612, v_{4}=0.5612, p_{4}=2.8$. The meshes are $200 \times 200$. The output time is $t=0.25$. The monitor function is $\omega=\sqrt{1+0.05|\bar{\nabla} e|^{2}}$.

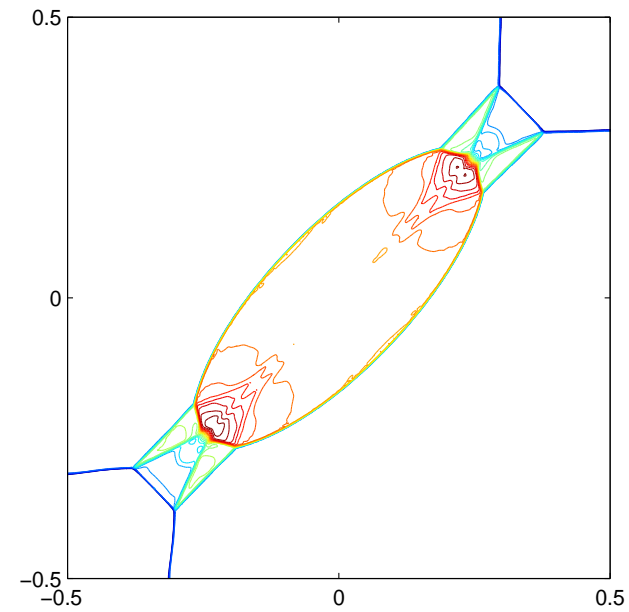

Figure 4.5: $\left[S_{12}^{+} S_{23}^{-} S_{34}^{+} S_{41}^{-}\right]$. The initial data are $\rho_{1}=1.4$, $u_{1}=-4.125, v_{1}=-4.125, p_{1}=1 ; \rho_{2}=8, u_{2}=4.125, v_{2}=$ $-4.125, p_{2}=116.5 ; \rho_{3}=1.4, u_{3}=4.125, v_{3}=4.125, p_{3}=1$; $\rho_{4}=8, u_{4}=-4.125, v_{4}=4.125, p_{4}=116.5$. The meshes are $100 \times 100$. The output time is $t=0.05$. The monitor function is $\omega=\sqrt{1+0.05|\bar{\nabla} e|^{2}}$.

\subsection{Interaction of pure vortex sheets (contact discontinuities)}

Contact discontinuities are discontinuous surfaces on which the flow is in the pressure equilibrium, no flow moving across them. We can describe them as the surfaces across which the pressure and the normal velocity component keep continuous but the density and tangential velocity component undergo (non-zero) jump. Hence there are two different types of contact discontinuities: One is the surface across which only the density undergoes a jump, and it behaves like a material surface; the other is the surface across which only the tangential surface has a jump and the vorticity becomes a delta-measure on it. We call the latter a vortex sheet that may be regarded as being composed of vortex filaments. The wings of an airplane are prototypical places to produce vortex sheets. The mathematical understanding of the evolution of vortex sheets is relatively well in incompressible fluid flows (see [58] for Wu's recent contributions and references therein), compared to that in (compressible) gas dynamics. In the present paper, we focus on the study of vortex sheets, although across them the density also has jumps. We provide two typical cases for the interactions of pure vortex sheets with themselves.

The case $J_{12}^{-} J_{23}^{-} J_{34}^{-} J_{41}^{-}$is the interaction of four vortex sheets with the same sign to form a spiral with the low density in its center, as shown in Figure 4.6. This is the typical cavitation phenomenon in gas dynamics. The interaction of infinite vortex sheets with the same sign is just Zhang-Zheng's explicit pattern in Appendix A.3. The case $J_{12}^{-} J_{23}^{+} J_{34}^{-} J_{41}^{+}$is totally different and displays the interaction of vortex sheets with different signs to produce infinite vortices, see Figure 4.7. It is worth pointing out that the concentration phenomenon of the density is observed in the formation process of vortices. These two configurations provide the fundamental building blocks for the formation of the spiral and vortices.

Since the density and the velocity undergo "big" jumps over the vortex sheets, it is natural that the monitor function in our scheme depends on these two variables: the density and velocity. The numerical results exhibits the perfect performance of the scheme in capturing spirals or small scale 
vortices, in comparison with the results by some other schemes, e.g. [15, 32, 43, 46].

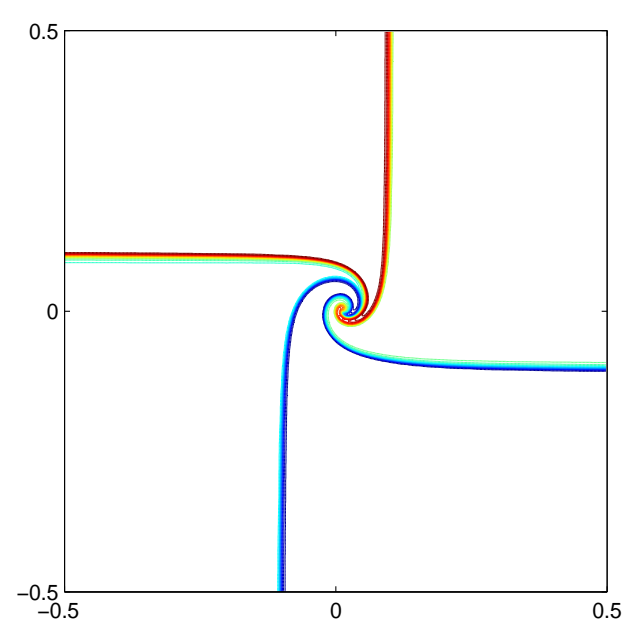

Figure 4.6: $\left[J_{12}^{-} J_{23}^{-} J_{34}^{-} J_{41}^{-}\right]$. The initial data are $\rho_{1}=$ $0.1, u_{1}=0.5, v_{1}=-0.5, p_{1}=10.0 ; \quad \rho_{2}=0.15, u_{2}=0.5, v_{2}=$ $0.5, p_{2}=10.0 ; \rho_{3}=0.09, u_{3}=-0.5, v_{3}=0.5, p_{3}=10.0 ; \rho_{4}=$ $0.05, u_{4}=-0.5, v_{4}=-0.5, p_{4}=10.0$. The meshes are $100 \times 100$. The output time is $t=0.2$. The monitor function is $\omega=$ $\sqrt{1+10\left(|\bar{\nabla} \rho|^{2}+|\bar{\nabla} u|^{2}+|\bar{\nabla} v|^{2}\right)}$.

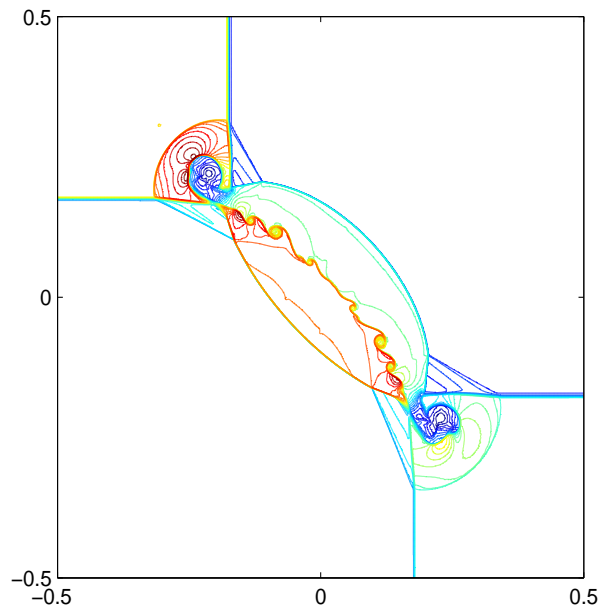

Figure 4.7: $\left[J_{12}^{-} J_{23}^{+} J_{34}^{-} J_{41}^{+}\right]$. The initial data are $\rho_{1}=0.5, u_{1}=$ $-0.5, v_{1}=-0.5, p_{1}=0.15 ; \rho_{2}=1.5, u_{2}=-0.5, v_{2}=0.5, p_{2}=$ $0.15 ; \rho_{3}=0.75, u_{3}=0.5, v_{3}=0.5, p_{3}=0.15 ; \quad \rho_{4}=1.0, u_{4}=$ $0.5, v_{4}=-0.5, p_{4}=0.15$. The meshes are $800 \times 800$. The output time is $t=0.35$. The monitor function is $\omega=$ $\sqrt{1+5\left(|\bar{\nabla} \rho|^{2}+|\bar{\nabla} u|^{2}+|\bar{\nabla} v|^{2}\right)}$.

\subsection{Interaction of planar shocks and vortex sheets}

The investigation of the interaction of the shocks and the vortex sheets is significant in practical applications, e.g. the environment of supersonic aircraft and missiles. There were numerous studies contributed to simulate the interaction of a planar shock with the cylindrical vortices (see [45] and references therein), and much attention was paid on the production and evolution of acoustic waves for the interaction with the weak shocks. In our current research the shock fronts are heavily distorted and the degree of distortion strongly depends on the relative strength of vortices and shocks. Hence linear theories depicting the acoustic waves are no longer valid here.

This group contains four families of subcases of the interaction of the shocks and the vortex sheets in Zhang-Zheng's four-wave Riemann problems. The first pair of interactions are shown in Figures 4.8 and 4.9 for $S_{12}^{+} J_{23}^{-} J_{34}^{+} S_{41}^{-}$. They are just the combination of the first and the second groups we showed previously: the interaction of the pure shocks and the interaction of the pure vortex sheets. The former exhibits the regular interaction of shocks and the latter the Mach-type interaction of the shocks; the vortex sheets with different signs interact independently first and then match the pattern resulting from the interaction of the shocks to form the global flow patterns.

The second pair in this group are shown in Figures 4.10 and 4.11 for $S_{12}^{-} J_{23}^{-} J_{34}^{+} S_{41}^{+}$. Unlike the case $S_{12}^{+} J_{23}^{-} J_{34}^{+} S_{41}^{-}$, the shocks and the vortex sheets interact each other directly for the present case to result in much more involved flow patterns. Figure 4.10 shows the interaction with relative small Mach number $M=0.3039$, the resultant pattern is of a regular reflection: the shock front $S_{12}^{+}$(resp. $S_{41}^{-}$) undergoes just a certain deformation as it interacts with the vortex sheet $J_{23}^{-}$(resp. $J_{34}^{+}$), and the vortex sheets interact with each other to form new vortices after their penetration through the shocks. As the strength of the shock increases, the flow pattern has dramatically changed, as shown 


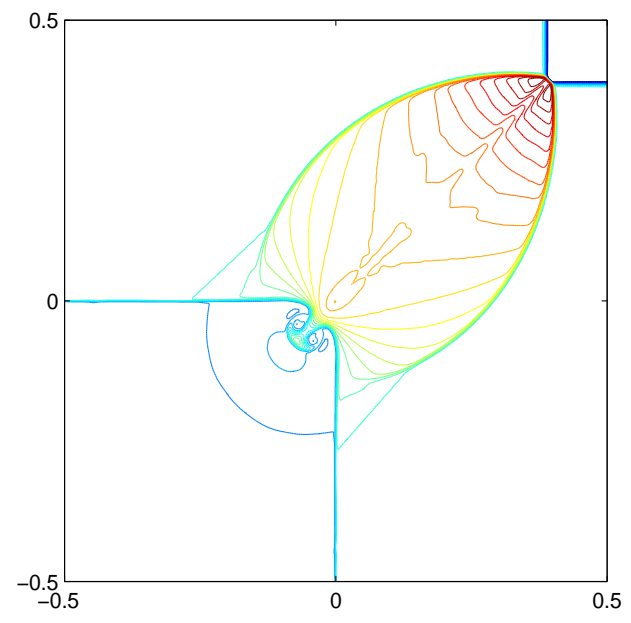

Figure 4.8: $\left[S_{12}^{+} J_{23}^{-} J_{34}^{+} S_{41}^{-}\right]$. The initial data are $\rho_{1}=$ $0.5313, u_{1}=0.0, v_{1}=0.0, p_{1}=0.4 ; \rho_{2}=1.0, u_{2}=0.7267, v_{2}=$ $0.0, p_{2}=1.0 ; \rho_{3}=0.8, u_{3}=0.0, v_{3}=0.0, p_{3}=1.0 ; \rho_{4}=1.0, u_{4}=$ $0.0, v_{4}=0.7276, p_{4}=1.0$. The meshes are $100 \times 100$. The output time is $t=0.25$. The monitor function is $\omega=$ $\sqrt{1+50\left(|\bar{\nabla} u|^{2}+|\bar{\nabla} v|^{2}\right)+10|\bar{\nabla} \rho|^{2}}$.

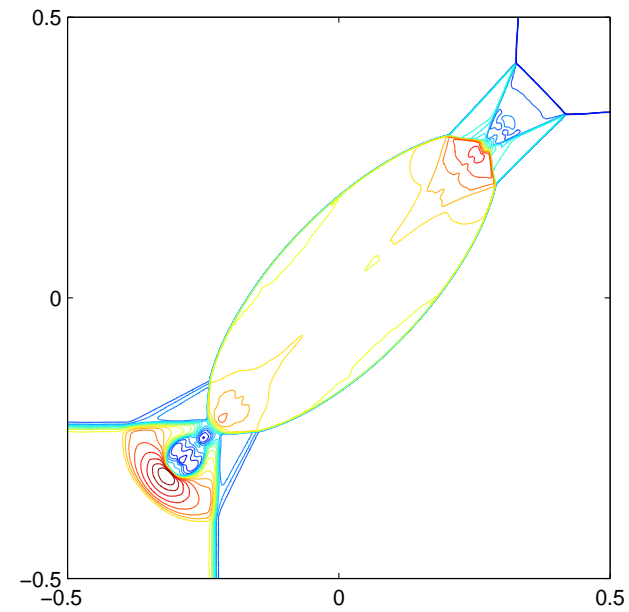

Figure 4.9: $\left[S_{12}^{+} J_{23}^{-} J_{34}^{+} S_{41}^{-}\right]$. The initial data are $\rho_{1}=$ $1.4, u_{1}=-4.125, v_{1}=-4.125, p_{1}=1.0, \rho_{2}=8.0, u_{2}=4.125, v_{2}=$ $-4.125, p_{2}=116.5, \rho_{3}=20.0, u_{3}=-4.125, v_{3}=-4.125, p_{3}=$ $116.5, \rho_{4}=8.0, u_{4}=-4.125, v_{4}=4.125, p_{4}=116.5$. The meshes are $200 \times 200$. The output time is $t=0.055$. The monitor function is $\omega=\sqrt{1+|\bar{\nabla} u|^{2}+|\bar{\nabla} v|^{2}+5|\bar{\nabla} \rho|^{2}}$.

in Figure 4.11 with Mach number $M=1.035$. The shocks experience diffraction and branching and then produce a complex cellular structure of the flow pattern.

In Figure 4.12 we show a different subcase $J_{12}^{-} S_{23}^{-} J_{34}^{-} S_{41}^{+}$of the shock-vortex interaction: $S_{41}^{+}$ diffracts at the interaction point with $J_{34}^{-}$to match $S_{23}^{-}$; while the vortex-sheet as the extension from $J_{34}^{-}$interact $J_{12}^{-}$to form a spiral. The situation becomes a little bit different for the case $J_{12}^{-} S_{23}^{-} J_{34}^{+} S_{41}^{+}$due to the different signs of $J_{12}^{-}$and $J_{34}^{+}$, as shown in Figure 4.13: there birth many small vortices. Moreover, the double Mach configuration of the shock reflection occurs in the flow pattern, which may be the consequence of the stronger shock waves.

\subsection{Interaction of pure planar rarefaction waves}

This group just involves the interaction of pure planar rarefaction waves, and it is the only available group for theoretical analysis up to now $[38,39]$. In the $1-\mathrm{D}$ case, the interaction of the simple waves or the rarefaction waves is relatively simple and they penetrate each other to form global continuous flow patterns, in which no shock or other singularities are newly developed. However, the situation becomes different for the interaction of the 2-D planar rarefaction waves.

There are two subcases in this group. We simulate the subcase $R_{12}^{+} R_{23}^{+} R_{34}^{-} R_{41}^{-}$and present the result in Figure 4.14, which seems to imply that the flow be smooth after the penetration through each other, similar to the 1-D case. However, a recent result [27] shows that insidious shocks may appear in the interaction region of the simple waves, depending on the angle between any two interacting planar rarefaction waves. The shocks are transonic, closely related to the Guderley phenomenon [31] in the steady counterpart from the viewpoint of the formation mechanism: the degeneracy of the flow on the sonic curves forces the simple waves to focus so that the shocks are produced from the envelope of the simple waves. This is a typical transonic flow pattern, deviating from the intuition motivated by $1-\mathrm{D}$ models. 


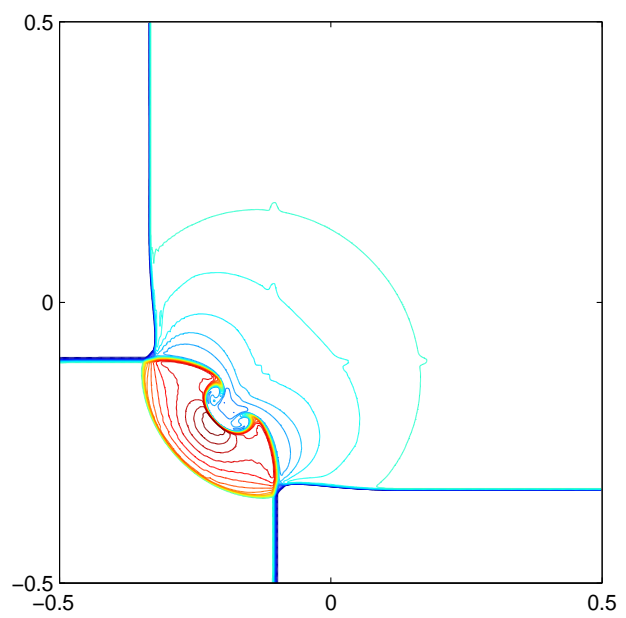

Figure 4.10: $\left[S_{12}^{-} J_{23}^{-} J_{34}^{+} S_{41}^{+}\right]$. The initial data are $\rho_{1}=1.0, u_{1}=$ $-0.3638, v_{1}=-0.3638, p_{1}=1.0, \rho_{2}=0.5313, u_{2}=0.3638, v_{2}=$ $-0.3638, p_{2}=0.4, \rho_{3}=1.0, u_{3}=-0.3638, v_{3}=-0.3638, p_{3}=0.4$, $\rho_{4}=0.5313, u_{4}=-0.3638, v_{4}=0.3638, p_{4}=0.4$. The mesh cells are $200 \times 200$. The output time is $t=0.28$. The monitor function is $\omega=\sqrt{1+50\left(|\bar{\nabla} \rho|^{2}+|\bar{\nabla} u|^{2}+|\bar{\nabla} v|^{2}\right)}$.

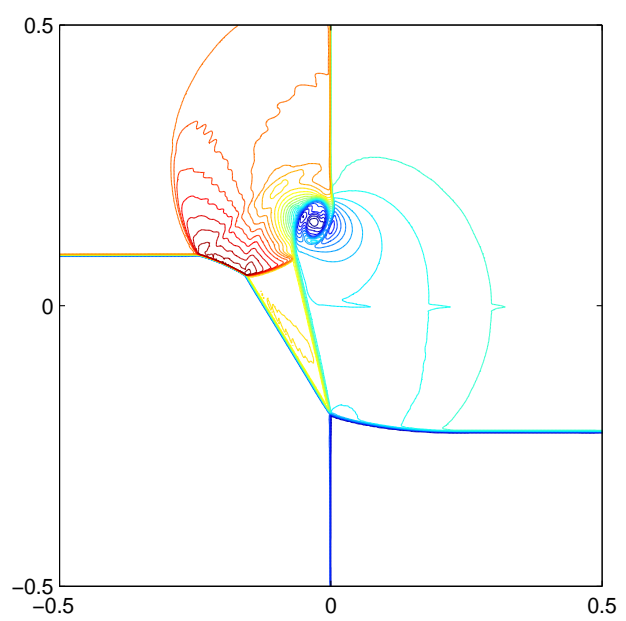

Figure 4.12: $\left[J_{12}^{-} S_{23}^{-} J_{34}^{-} S_{41}^{+}\right]$. The initial data are $\rho_{1}=$ $1.0, u_{1}=0.0, v_{1}=0.0, p_{1}=1.0 ; \rho_{2}=1.4, u_{2}=0.0, v_{2}=1.0, p_{2}=$ 1.0; $\rho_{3}=0.7, u_{3}=0.0, v_{3}=1.6742, p_{3}=0.3636 ; \rho_{4}=0.5, u_{4}=$ $0.0, v_{4}=0.7978, p_{4}=0.3636$. The mesh cells are $100 \times 100$. The output time is $t=0.28$. The monitor function is $\omega=$ $\sqrt{1+40.0\left(|\bar{\nabla} \rho|^{2}+|\bar{\nabla} u|^{2}+|\bar{\nabla} v|^{2}\right)}$.

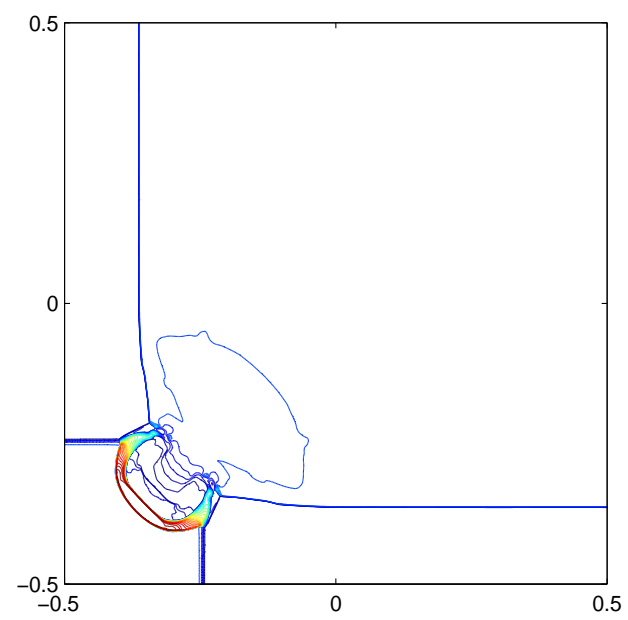

Figure 4.11: $\left[S_{12}^{-} J_{23}^{-} J_{34}^{+} S_{41}^{+}\right]$. The initial data are $\rho_{1}=$ $7.039, u_{1}=-2.037, v_{1}=-2.037, p_{1}=30.0, \quad \rho_{2}=1.4, u_{2}=$ $2.037, v_{2}=-2.037, p_{2}=1.0, \quad \rho_{3}=7.039, u_{3}=-2.037, v_{3}=$ $-2.037, p_{3}=1.0, \rho_{4}=1.4, u_{4}=-2.037, v_{4}=2.037, p_{4}=1.0$. The mesh cells are $400 \times 400$. The output time is $t=0.119$. The monitor function is $\omega=\sqrt{1+5.0\left(|\bar{\nabla} \rho|^{2}+|\bar{\nabla} u|^{2}+|\bar{\nabla} v|^{2}\right)}$.

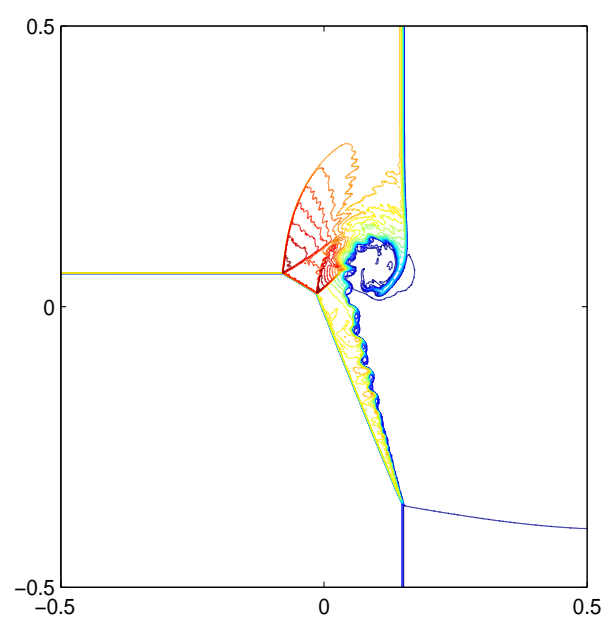

Figure 4.13: $\left[J_{12}^{-} S_{23}^{-} J_{34}^{+} S_{41}^{+}\right]$. The initial data are $\rho_{1}=$ $2.04, u_{1}=1.0, v_{1}=-1.0, p_{1}=15.0 ; \rho_{2}=15.0, u_{2}=1.0, v_{2}=$ $0.0, p_{2}=15.0 ; \quad \rho_{3}=4.4022, u_{3}=1.0, v_{3}=2.4444, p_{3}=2.0 ;$ $\rho_{4}=0.5870, u_{4}=1.0, v_{4}=2.9558, p_{4}=2.0 . \quad$ The mesh cells are $1200 \times 1200$. The output time is $t=0.15$. The monitor function is $\omega=\sqrt{1+0.01\left(|\bar{\nabla} \rho|^{2}+|\bar{\nabla} u|^{2}+|\bar{\nabla} v|^{2}\right)}$. 


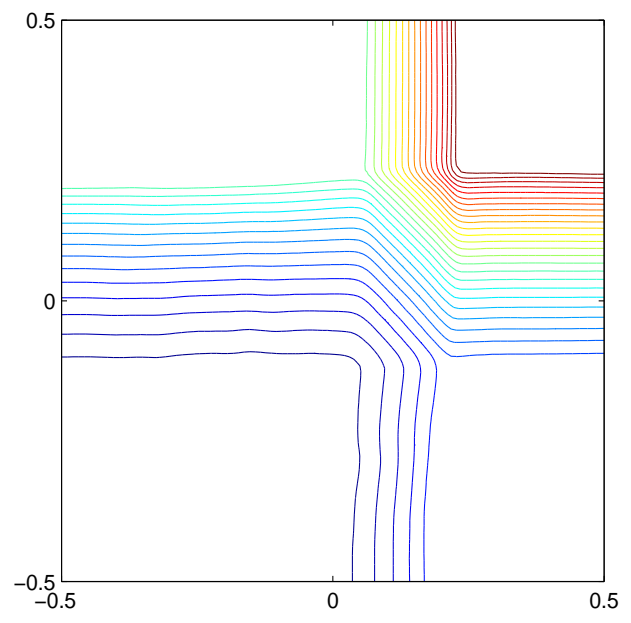

Figure 4.14: $\left[R_{12}^{+} R_{23}^{+} R_{34}^{-} R_{41}^{-}\right]$. The initial data are $\rho_{1}=$ $1.0, u_{1}=0.0, v_{1}=0.0, p_{1}=1.0 ; \rho_{2}=0.5179, u_{2}=-0.7259, v_{2}=$ $0.0, p_{2}=0.4 ; \quad \rho_{3}=0.1072, u_{3}=-0.7259, v_{3}=-1.4045, p_{3}=$ $0.0439 ; \rho_{4}=0.2579, u_{4}=0.0, v_{4}=-1.4045, p_{4}=0.15$. The mesh cells are $100 \times 100$. The output time is $t=0.2$. The

monitor function is $\omega=\sqrt{1+100\left(|\bar{\nabla} u|^{2}+|\bar{\nabla} v|^{2}+|\bar{\nabla} \rho|^{2}\right)}$.

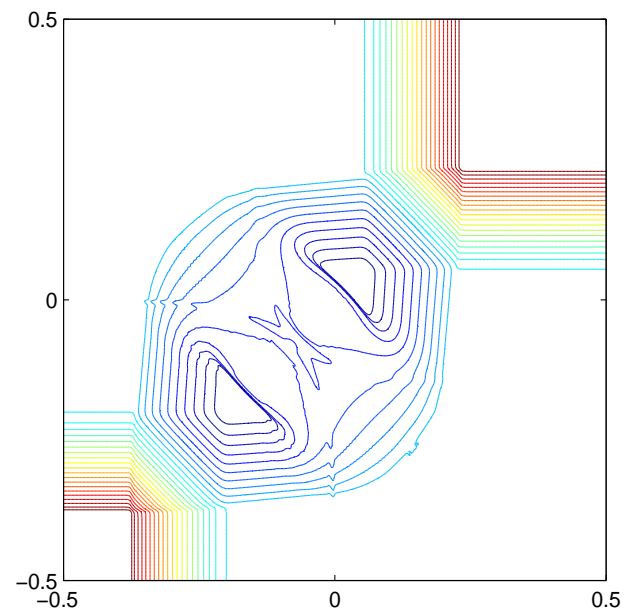

Figure 4.15: $\left[R_{12}^{+} R_{23}^{-} R_{34}^{+} R_{41}^{-}\right]$. The initial data are $\rho_{1}=$ $1.0, u_{1}=0.0, v_{1}=0.0, p_{1}=1.0 ; \rho_{2}=0.5197, u_{2}=-0.7259, v_{2}=$ $0.0, p_{2}=0.4 ; \quad \rho_{3}=1.0, u_{3}=-0.7259, v_{3}=-0.7259, p_{3}=1.0$; $\rho_{4}=0.5179, u_{4}=0.0, v_{4}=-0.7259, p_{4}=0.4$. The mesh cells are $100 \times 100$. The output time is $t=0.2$. The monitor function is $\omega=\sqrt{1+100\left(|\bar{\nabla} u|^{2}+|\bar{\nabla} v|^{2}+|\bar{\nabla} \rho|^{2}\right)}$.

Figure 4.15 displays the simulation for the symmetric case $R_{12}^{+} R_{23}^{-} R_{34}^{+} R_{41}^{-}$. We can see that there are two symmetric (transonic) shocks in the interaction region after the mutual penetration of the planar rarefaction waves. This is easily understood since the symmetric axis $x / t-u_{1}=y / t-v_{1}$ can be regarded as a rigid wall and the rarefaction waves will be compressed when approaching it. However, we have found that there may be a vacuum bubble in the central area of interaction region as the planar rarefaction waves are strong enough [39].

These two cases were ever thought to be the most accessible theoretically. From both the simulations pursued above and the theorems established in $[27,39]$, it is realized that there are plenty of interesting flow patterns far beyond the intuition and the understanding in our database.

\subsection{Interaction of rarefaction waves and vortex sheets}

In Figures 4.16-4.20 it is to simulate the group of the interactions of the rarefaction waves and the vortex-sheets. The flow configurations look relatively simple compared to other groups in the preceding subsections. The vortex-sheets are just slightly affected and distorted when penetrating rarefaction waves since they are continuous.

Figure 4.16 displays the simulation for $R_{12}^{+} J_{23}^{+} J_{34}^{-} R_{41}^{-}$. It is clear to see how the vortex-sheets interact the rarefaction waves. Due to the different signs of $J_{23}^{+}$and $J_{34}^{-}$, many small scale vortices are formed, just like the case $J_{12}^{-} J_{23}^{+} J_{34}^{-} J_{41}^{+}$. Figure 4.17 shows close-up of the vortices in the interaction region. It is worth pointing out that much finer meshes are necessary to capture small scale vortices. Due to this, $800 \times 800$ meshes are used in our simulation. In Figures $4.18-4.20$ we continue to simulate the other cases. We observe an incipient instability of vortex sheets in Figures 4.19 and 4.20, for which we therefore use finer meshes. 


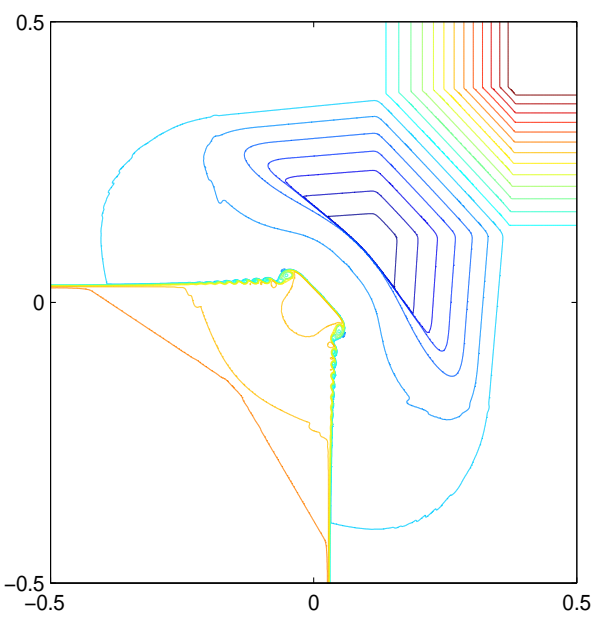

Figure 4.16: $\left[R_{12}^{+} J_{23}^{+} J_{34}^{-} R_{41}^{-}\right]$. The initial data are $\rho_{1}=$ $1.0, u_{1}=0.1, v_{1}=0.1, p_{1}=1.0 ; \rho_{2}=0.5197, u_{2}=-0.6259, v_{2}=$ $0.1, p_{2}=0.4 ; \rho_{3}=0.8, u_{3}=0.1, v_{3}=0.1, p_{3}=0.4 ; \rho_{4}=0.5197, u_{4}=$ $0.1, v_{4}=-0.6259, p_{4}=0.4$. The mesh cells are $800 \times 800$. The output time is $t=0.3$. The monitor function is $\omega=$ $\sqrt{1+200\left(|\bar{\nabla} u|^{2}+|\bar{\nabla} v|^{2}+|\bar{\nabla} \rho|^{2}\right)}$.

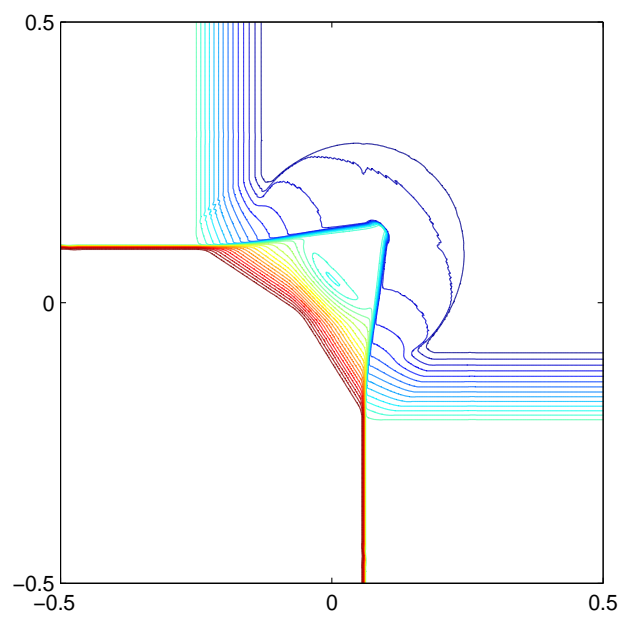

Figure 4.18: $\left[R_{12}^{-} J_{23}^{+} J_{34}^{-} R_{41}^{+}\right]$. The initial data are $\rho_{1}=$ $0.5686, u_{1}=0.3, v_{1}=0.5, p_{1}=0.3302 ; \rho_{2}=1.0, u_{2}=-0.244, v_{2}=$ $0.5, p_{2}=0.7279 ; \rho_{3}=1.5, u_{3}=0.3, v_{3}=0.5, p_{3}=0.7279 ; \rho_{4}=$ $1.0, u_{4}=0.3, v_{4}=-0.0389, p_{4}=0.7279$. The mesh cells are $100 \times 100$. The output time is $t=0.2$. The monitor function is $\omega=\sqrt{1+200\left(|\bar{\nabla} u|^{2}+|\bar{\nabla} v|^{2}+|\bar{\nabla} \rho|^{2}\right)}$.

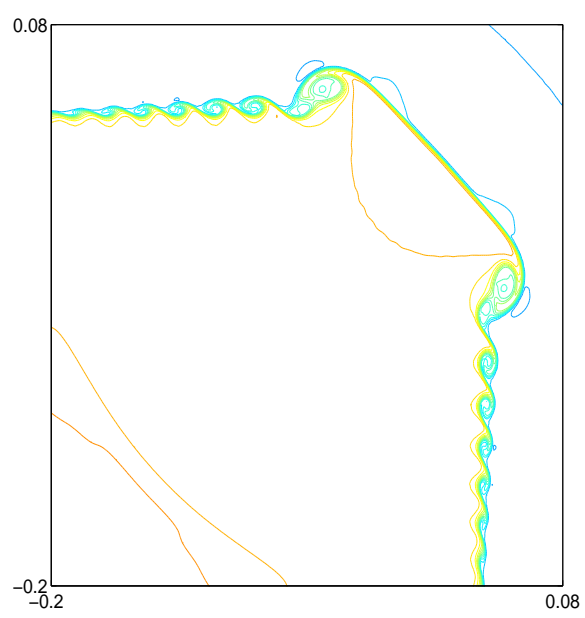

Figure 4.17: Local enlargement of Figure 4.16 for vortices.

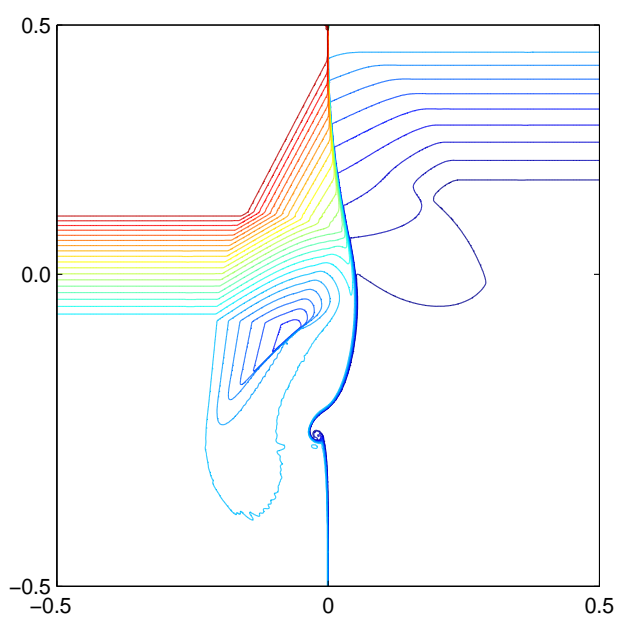

Figure 4.19: $\left[J_{12}^{+} R_{23}^{+} J_{34}^{+} R_{41}^{-}\right]$. The initial data are $\rho_{1}=$ $1.0, u_{1}=0.0, v_{1}=0.3, p_{1}=1.0 ; \rho_{2}=2.0, u_{2}=0.0, v_{2}=-0.3, p_{2}=$ $1.0 ; \rho_{3}=1.039, u_{3}=0.0, v_{3}=-0.8133, p_{3}=0.4 ; \rho_{4}=0.5197, u_{4}=$ $0.0, v_{4}=-0.4259, p_{4}=0.4$. The mesh cells are $800 \times 800$. The output time is $t=0.3$. The monitor function is $\omega=$ $\sqrt{1+1.0\left(|\bar{\nabla} u|^{2}+|\bar{\nabla} v|^{2}+|\bar{\nabla} \rho|^{2}\right)}$. 


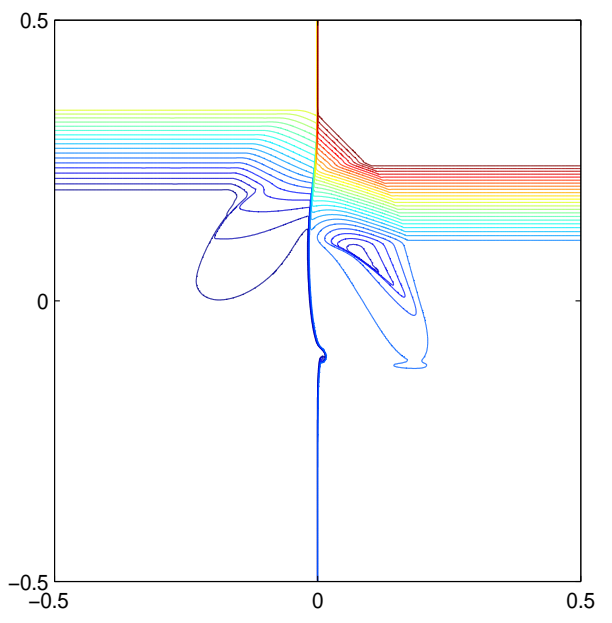

Figure 4.20: $\left[J_{12}^{+} R_{23}^{+} J_{34}^{-} R_{41}^{-}\right]$. The initial data are $d 1=$ $1.5, u 1=0.0, v 1=0.0, p 1=2.0 ; d 2=1.2, u 2=0.0, v 2=0.4, p 2=2.0$; $d 3=0.731408, u 3=0.0, v 3=-0.32, p 3=1.0 ; d 4=0.91426, u 4=$ $0.0, v 4=-0.64403, p 4=1.0$. The mesh cells are $800 \times 800$. The output time is $t=0.18$. The monitor function is $\omega=$ $\sqrt{1+1.0\left(|\bar{\nabla} u|^{2}+|\bar{\nabla} v|^{2}+|\bar{\nabla} \rho|^{2}\right)}$.

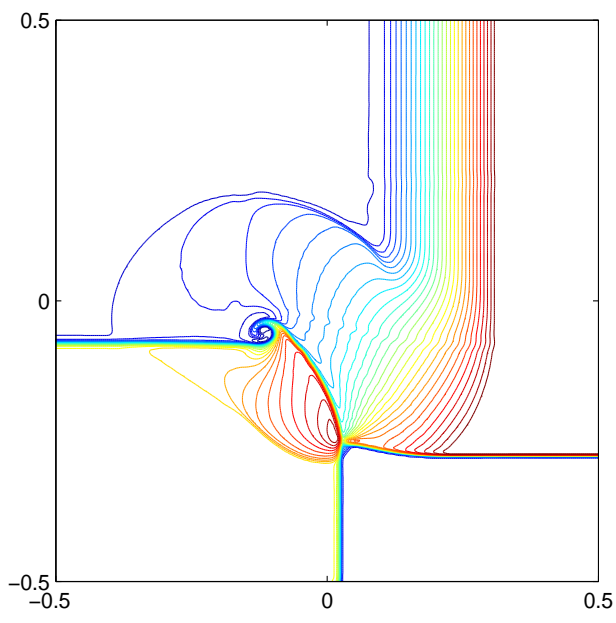

Figure 4.21: $\left[R_{12}^{+} J_{23}^{+} J_{34}^{+} S_{41}^{+}\right]$. The initial data are $\rho_{1}=$ $1.0, u_{1}=0.1, v_{1}=-0.3, p_{1}=1.0 ; \rho_{2}=0.5179, u_{2}=-0.6259, v_{2}=$ $-0.3, p_{2}=0.4 ; \quad \rho_{3}=0.8, u_{3}=0.1, v_{3}=-0.3, p_{3}=0.4 ; \quad \rho_{4}=$ $0.5313, u_{4}=0.1, v_{4}=0.4276, p_{4}=0.4$. The mesh cells are $300 \times$ 300. The output time is $t=0.2$. The monitor function is $\omega=\sqrt{1+0.1\left(|\bar{\nabla} u|^{2}+|\bar{\nabla} v|^{2}+|\bar{\nabla} \rho|^{2}\right)}$.

\subsection{Interaction of shocks, rarefaction waves and vortex sheets}

In Figures 4.21-4.25, we collect the interaction of rarefaction waves, shocks and vortex sheets in this group. All of them are clearly simulated with $200 \times 200$ meshes. The numerical results are basically the combination of local wave patterns in the preceding groups: Spirals, vortices, the compression of rarefaction waves etc.

\section{Discussion}

In [25] the GRP method was combined with the adaptive technique to derive the adaptive GRP scheme. We showed the capability of this scheme in overcoming difficulties such as the start-up error for a single shock, and the numerical instability of the almost stationary shock and displayed some performance of the CPU time and the simulation of several 2-D benchmark problems. In this paper, we continue the program and mainly investigate the properties of the $2-\mathrm{D}$ version. Precisely, two main aspects are discussed:

1. Numerical accuracy. To access the accuracy of this scheme numerically, we provide the explicit formulae of the exact solutions of four examples, two of them being rarely available from the recent theoretical works $[34,38,61]$. Then we make the comparison to address the accuracy: For the single oblique rarefaction wave case, the accuracy can attain second order, while for the shock case it is just of first order and for the other two cases of continuous solutions the orders are slightly more than one and half. Despite lack of a super-convergence property, the accuracy is still within our expectation.

2. Simulation of 2-D complex flow configurations. We choose Zhang-Zheng's four-wave Riemann problems for 2-D compressible Euler equations to demonstrate the performance 


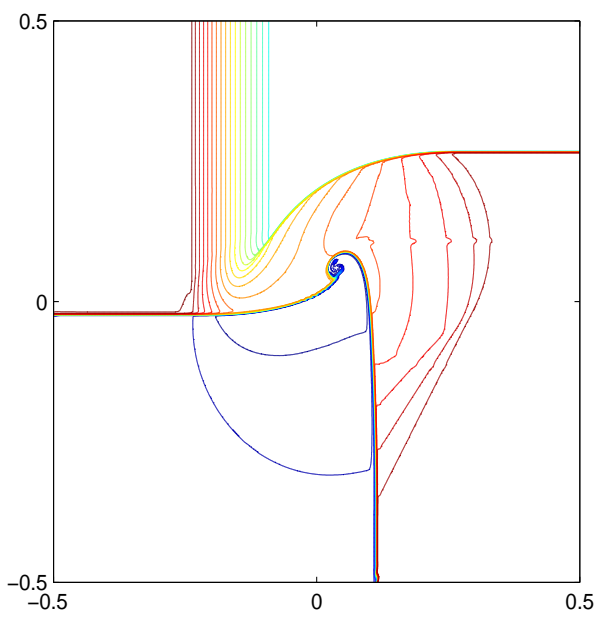

Figure 4.22: $\left[R_{12}^{-} J_{23}^{+} J_{34}^{+} S_{41}^{-}\right]$. The initial data are $d 1=1.5, u 1=0.5, v 1=-0.1, p 1=0.8 ; \quad d 2=2.7824, u 2=$ $-0.06828, v 2=-0.1, p 2=1.9 ; d 3=0.9, u 3=0.5, v 3=-0.1, p 3=$ $1.9 ; d 4=2.7313, u 4=0.5, v 4=0.4750, p 4=1.9$. The mesh cells are $400 \times 400$. The output time is $t=0.2$. The monitor function is $\omega=\sqrt{1+50\left(|\bar{\nabla} u|^{2}+|\bar{\nabla} v|^{2}\right)+10|\bar{\nabla} \rho|^{2}}$.

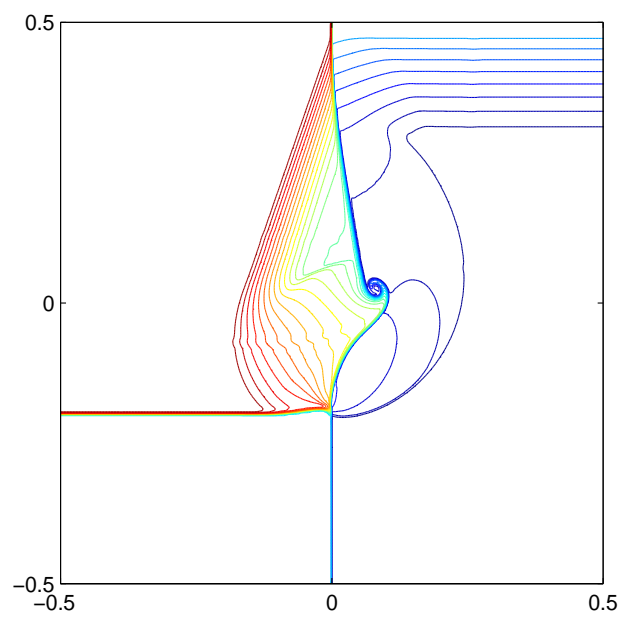

Figure 4.24: $\left[J_{12}^{+} S_{23}^{-} J_{34}^{+} R_{41}^{+}\right]$. The initial data are $d 1=$ $1.0, u 1=0.0, v 1=1.0, p 1=1.0 ; d 2=2.0, u 2=0.0, v 2=-0.3, p 2=$ $1.0 ; d 3=1.0625, u 3=0.0, v 3=0.2145, p 3=0.4 ; d 4=0.5179, u 4=$ $0.0, v 4=0.2741, p 4=0.4$. The mesh cells are $200 \times 200$. The output time is $t=0.2$. The monitor function is $\omega=$ $\sqrt{1+50\left(|\bar{\nabla} u|^{2}+|\bar{\nabla} v|^{2}\right)+10|\bar{\nabla} \rho|^{2}}$.

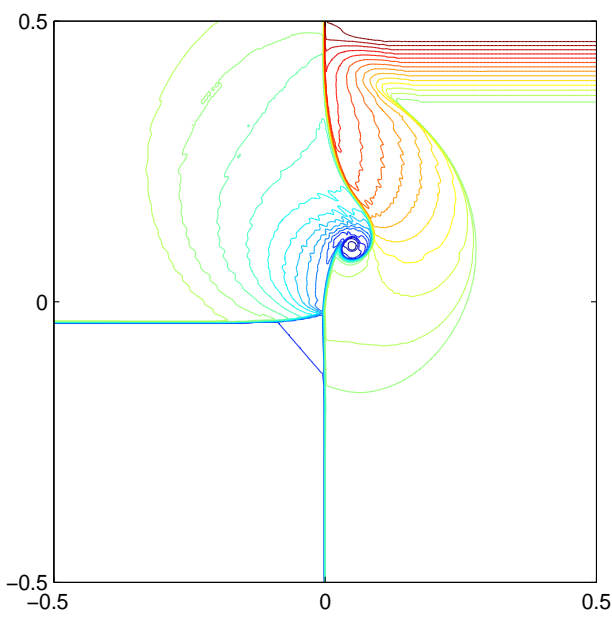

Figure 4.23: $\left[J_{12}^{-} S_{23}^{-} J_{34}^{-} R_{41}^{-}\right]$. The initial data are $\rho_{1}=$ $1.2, u_{1}=0.0, v_{1}=0.6, p_{1}=0.8 ; \rho_{2}=0.9, u_{2}=0.0, v_{2}=0.8, p_{2}=$ $0.8 ; \quad \rho_{3}=0.645283, u_{3}=0.0, v_{3}=1.162738, p_{3}=0.5 ; \quad \rho_{4}=$ $0.857791, u_{4}=0.0, v_{4}=0.286315, p_{4}=0.5$. The mesh cells are $200 \times 200$. The output time is $t=0.3$. The monitor function is $\omega=\sqrt{1+50\left(|\bar{\nabla} u|^{2}+|\bar{\nabla} v|^{2}\right)+10|\bar{\nabla} \rho|^{2}}$.

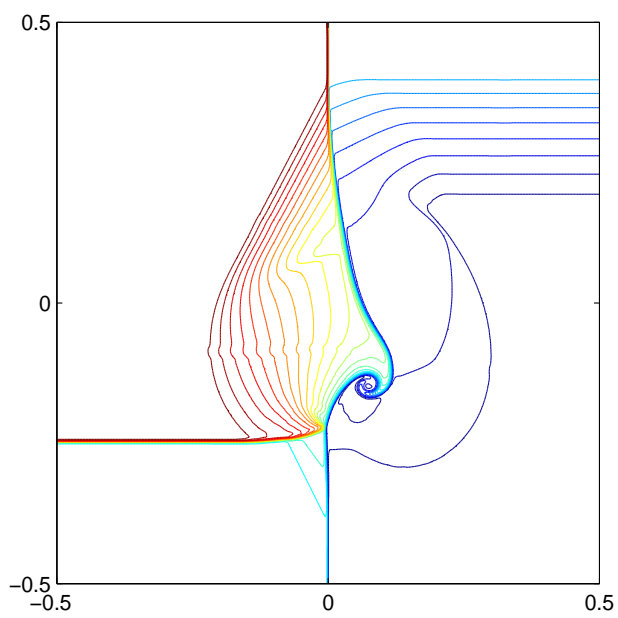

Figure 4.25: $\left[J_{12}^{+} S_{23}^{-} J_{34}^{-} R_{41}^{+}\right]$. The initial data are $\rho_{1}=$ $1.0, u_{1}=0.0, v_{1}=0.3, p_{1}=1.0 ; \rho_{2}=2.0, u_{2}=0.0, v_{2}=-0.3, p_{2}=$ $1.0 ; \rho_{3}=1.0625, u_{3}=0.0, v_{3}=0.2145, p_{3}=0.4 ; \rho_{4}=0.5179, u_{4}=$ $0.0, v_{4}=-0.4259, p_{4}=0.4$. The mesh cells are $200 \times 200$. The output time is $t=0.26$. The monitor function is $\omega=$ $\sqrt{1+50\left(|\bar{\nabla} u|^{2}+|\bar{\nabla} v|^{2}\right)+10|\bar{\nabla} \rho|^{2}}$. 
of this scheme in capturing 2-D complex wave configurations. Compared to those by other friendly-used schemes $[15,32,43,46]$, the adaptive GRP scheme presents quite well results. In particular, it can capture the structure of the spiral formation and even small scale vortices, see Figures 4.6,4.7, 4.16 and 4.17.

As a byproduct, we have tried to construct the monitor function as uniformly as possible. Conclusions are that if only shocks are involved in the computation, the internal energy is a good candidate in the construction; however, once there present vortex sheets in the solutions, it is more plausible to use the density and the velocity to construct the monitor function. This is reasonable because the internal energy (equivalently the entropy) plays fundamental role for shocks and the density (resp. the tangential velocity) undergo big "jumps" through the vortex sheets.

\section{A Set-up of the 2-D Riemann problem}

The 2-D Riemann problem for (2.1) is a special class of initial value problems subject to the radially invariant initial data

$$
U(x, y, 0)=U_{0}(\theta), \quad \theta=\arctan (y / x) .
$$

The value $U_{0}(\theta)$ is often taken to be several pieces of sectorial constant data in the form

$$
U_{0}(\theta)=U_{i}, \quad \theta_{i}<\theta<\theta_{i+1},
$$

where $i=1, \cdots, k, \theta_{k+1}=\theta_{1}+2 \pi$. Such data have two kinds of discontinuities: the rays separating $U_{i}$ and $U_{i+1}$, and the origin on which all rays focus. In particular, a four-wave Riemann problem was formulated and solution structures were conjectured in [60], for which the initial data is constant in each quadrant.

A primary approach to solve 2-D Riemann problem analytically is first to take dimension reduction via a self-similar transformation and then study the resulting problem in the self-similar plane-the $(x / t, y / t)$ plane, i.e., the solution has the property $U(x, y, t)=U(\xi, \eta, 1),(\xi, \eta)=(x / t, y / t)$. Then (2.1) becomes

$$
(-\xi U-F(U))_{\xi}+(-\eta U-G(U))_{\eta}=-2 U .
$$

For most cases, the 2-D Riemann problem has not been solved theoretically due to inherent challenges such as transonic flow problems, except the following several cases, for which we are even able to provide explicit formulae so that numerical solutions can be compared with them. These explicit solutions, to some extent, can be used to be benchmark problems to test the accuracy of multi-dimensional numerical schemes.

\section{A.1 1-D planar waves}

Given a direction $(\mu, \nu)$ with $\mu^{2}+\nu^{2}=1$, we prescribe the initial data for one dimensional planar waves as two pieces of constant states

$$
(\rho, u, v, p)(x, y, 0)= \begin{cases}\left(\rho_{i}, u_{i}, v_{i}, p_{i}\right), & \mu x+\nu y>0, \\ \left(\rho_{j}, u_{j}, v_{j}, p_{j}\right), & \mu x+\nu y<0,\end{cases}
$$

for which the $(\mu, \nu)$ is oriented from the state $\left(\rho_{j}, u_{j}, v_{j}, p_{j}\right)$ to the state $\left(\rho_{i}, u_{i}, v_{i}, p_{i}\right)$. We denote by $\tilde{u}:=\mu u+\nu v$ the velocity component normal to the discontinuity plane and by $\tilde{v}:=-\nu u+\mu v$ 
the tangent velocity component. The problem (2.1)-(A.4) can be regarded in the way that we rotate the one dimensional Riemann problem along the $x$-direction with an appropriate angle in a counter-clockwise manner. The one-dimensional planar waves include planar rarefaction waves, planar shocks and planar contact discontinuities (vortex sheets). For the simplicity of presentation we denote $\zeta=(\mu x+\nu y) / t$ below.

(i) A rarefaction wave. A rarefaction wave is a fan that spans from the state $\left(\rho_{i}, u_{i}, v_{i}, p_{i}\right)$ to the state $\left(\rho_{j}, u_{j}, v_{j}, p_{j}\right)$, and these two states satisfy,

$$
R_{i j}^{ \pm}: \quad \zeta=\tilde{u}+c, \quad c=\gamma p / \rho, \quad p \rho^{-\gamma}=p_{i} \rho_{i}^{-\gamma}=p_{j} \rho_{j}^{-\gamma}, \quad \tilde{u}=\tilde{u}_{i} \pm \frac{2}{\gamma-1}\left(c-c_{i}\right), \quad \tilde{v}=\tilde{v}_{i}=\tilde{v}_{j},
$$

where $0 \leq \rho_{j} \leq \rho \leq \rho_{i}$ for "+" sign, or $0 \leq \rho_{i} \leq \rho \leq \leq \rho_{j}$ for "-" sign.

(ii) A shock wave. A shock wave is defined using the Rankine-Hugoniot relation to separate two states $\left(\rho_{i}, u_{i}, v_{i}, p_{i}\right)$ and $\left(\rho_{j}, u_{j}, v_{j}, p_{j}\right)$,

$$
S_{i j}^{ \pm}: \quad \zeta=\tilde{u} \pm \sqrt{\frac{\rho_{i}\left(p_{i}-p_{j}\right)}{\rho_{j}\left(\rho_{i}-\rho_{j}\right)}}, \frac{\tilde{u}_{i}-\tilde{u}_{j}}{\rho_{i}-\rho_{j}}= \pm \sqrt{\frac{p_{i}-p_{j}}{\rho_{i} \rho_{j}\left(\rho_{i}-\rho_{j}\right)}}, \quad \tilde{v}=\tilde{v}_{j}=\tilde{v}_{i}, \quad \frac{p_{i}}{p_{j}}=\frac{\rho_{i}-\pi^{2} \rho_{j}}{\rho_{j}-\pi^{2} \rho_{i}},
$$

where $\pi^{2}=\frac{\gamma-1}{\gamma+1}$, and the entropy condition reads

$$
p_{i}<p_{j} \text { for "+" sign or } p_{i}>p_{j} \text { for "-" sign. }
$$

(iii) A contact discontinuity. The states $\left(\rho_{i}, u_{i}, v_{i}, p_{i}\right)$ and $\left(\rho_{j}, u_{j}, v_{j}, p_{j}\right)$ that are separated by a contact discontinuity should satisfy

$$
J_{i j}^{ \pm}: \quad \zeta=\tilde{u}_{i}=\tilde{u}_{j}, \quad p_{i}=p_{j},
$$

where the signs “ \pm ” are determined by the curl of velocity field on the discontinuity,

$$
\operatorname{curl}(u, v)=v_{x}-u_{y}= \pm \infty .
$$

Just as we previously mentioned across this discontinuity the density $\rho$ or/and the tangent velocity component $\tilde{v}$ undergoes a jump.

Note that across this discontinuity the density $\rho$ or/and the tangent velocity component $\tilde{v}$ undergoes a jump. If only the density $\rho$ undergoes a jump, this discontinuity behaves just like a surface separating to two different materials. In contrast, if only $\tilde{v}$ undergoes a jump, it is a vortex sheet. In the present paper, we use the terminology "vortex sheet" to mean a discontinuity across which the tangential velocity component $\tilde{v}$ is discontinuous with a possible jump of the density $\rho$.

\section{A.2 The collapse problem of a wedge-shaped dam}

The collapse problem of a wedge-shaped dam is hydraulically classical and considered to be a special case of the 2-D Riemann problem. It is the only case that is solved thoroughly up to now. See $[34,38]$

This problem is formulated as follows. The isentropic form of governing equation (2.1) is taken here, for which $p=\rho^{\gamma}$. For the shallow water case, $\gamma=2$ and $\rho$ can be regarded as the height of 


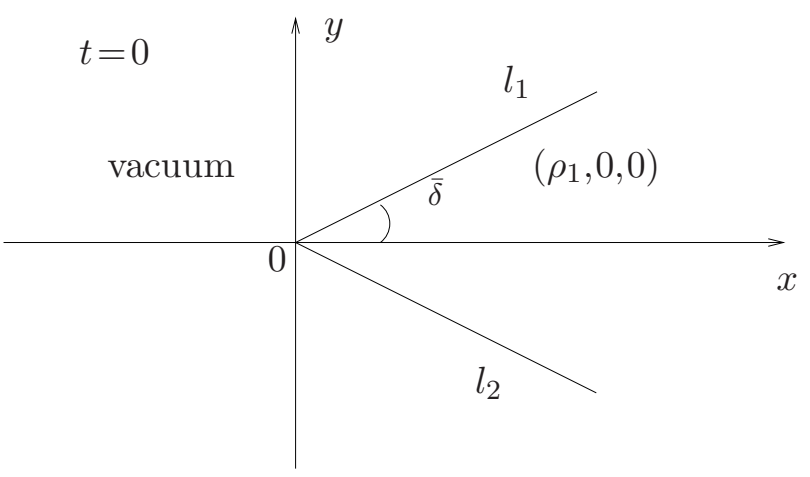

(a) Initial data

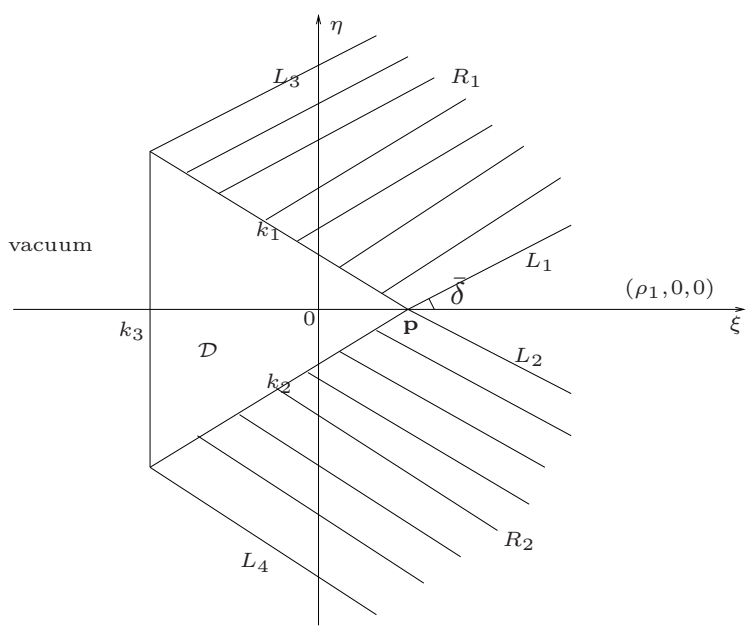

(b) Interaction of rarefaction waves

Figure A.1: The expansion of wedge of gas.

water from the bottom. We impose the initial data as

$$
(\rho, u, v)(x, y, 0)= \begin{cases}\left(\rho_{0}, 0,0\right), & -\bar{\delta}<\theta<\bar{\delta} \\ (0, \bar{u}, \bar{v}), & \text { otherwise }\end{cases}
$$

where $\rho_{0}>0,(\bar{u}, \bar{v})$ is the velocity of wave front, not being specified in the vacuum interface, $\theta=\arctan \left(\frac{y}{x}\right)$ is the polar angle, and $\bar{\delta}$ is the half angle of the wedge restricted between 0 and $\frac{\pi}{2}$. Away from the sharp corner the gas expands into the vacuum as planar rarefaction waves $R_{1}$ and $R_{2}$ of the form $(\rho, u, v)(\zeta)$,

$$
(\rho, u, v)(x, y, t)= \begin{cases}\left(\rho_{1}, 0,0\right), & \zeta_{k}>1, \\ (\rho, u, v)(\zeta), & -\frac{2}{\gamma-1} \leq \zeta_{k} \leq 1 \\ \text { vacuum, } & \zeta_{k}<-\frac{2}{\gamma-1}\end{cases}
$$

where $\zeta_{k}=\left(\mu_{k} x+\nu_{k} y\right) / t, k=1,2$, for $R_{1}$ and $R_{2}$, and $\rho_{1}$ is so normalized that $c_{1}^{2}=\gamma \rho_{1}^{\gamma-1}=1$. Denote by $\left(\mu_{1}, \nu_{1}\right)=(\sin \bar{\delta},-\cos \bar{\delta}),\left(\mu_{2}, \nu_{2}\right)=(\sin \bar{\delta}, \cos \bar{\delta})$ the normal directions of the initial discontinuities $l_{1}: x \sin \bar{\delta}-y \cos \bar{\delta}=0$ and $l_{2}: x \sin \bar{\delta}+y \cos \bar{\delta}=0, x>0$, respectively. The rarefaction waves $R_{1}$ and $R_{2}$ begin to interact at $P=\left(\frac{1}{\sin \delta}, 0\right)$ in the $(\xi, \eta)$ plane due to the presence of sharp corner and a wave interaction region $\mathcal{D}$ forms to separate from the planar rarefaction waves by two characteristics $k_{1}$, $k_{2}$. Then the solution consists of five patches: the interaction region $\mathcal{D}$, the constant state $\left(\rho_{1}, 0,0\right)$, the vacuum region, and the planar rarefaction waves $R_{1}$ and $R_{2}$.

It was shown in [34] that as the wedge angle $\bar{\delta}$ and the polytropic index $\gamma$ are related with the formula $\tan ^{2} \bar{\delta}=\frac{3-\gamma}{\gamma+1}$ for $1<\gamma<3$, the solution, particularly in the wave interaction region $\mathcal{D}$, can 
be written out explicitly. The two characteristics $k_{1}, k_{2}$ are expressed as,

$$
\begin{aligned}
& k_{1}: \quad \sqrt{(3-\gamma)(\gamma+1)} \eta_{1}=(\gamma-1) \xi_{1}+2, \quad\left(\eta_{1}>0,-\frac{2}{\gamma-1} \leq \xi_{1} \leq 1\right), \\
& k_{2}: \quad-\sqrt{(3-\gamma)(\gamma+1)} \eta_{2}=(\gamma-1) \xi_{2}+2, \quad\left(\eta_{2}<0,-\frac{2}{\gamma-1} \leq \xi_{2} \leq 1\right),
\end{aligned}
$$

where $\left(\xi_{1}, \eta_{1}\right)=(\xi \sin \bar{\delta}-\eta \cos \bar{\delta}, \xi \cos \bar{\delta}+\eta \sin \bar{\delta})$ and $\left(\xi_{2}, \eta_{2}\right)=(\xi \sin \bar{\delta}+\eta \cos \bar{\delta},-\xi \cos \bar{\delta}+\eta \sin \bar{\delta})$. The vacuum interface is $\xi=-\frac{2 \sin \bar{\delta}}{\gamma-1}$. The five patches are now expressed as:

(i) As $\xi \sin \bar{\delta}-\eta \cos \bar{\delta}>1$ and $\xi \sin \bar{\delta}+\eta \cos \bar{\delta}>1$, the solution is the constant state at rest,

$$
(\rho, u, v)(\xi, \eta)=\left(\rho_{1}, 0,0\right) .
$$

(ii) As $-\frac{2}{\gamma-1} \leq \xi \sin \bar{\delta}-\eta \cos \bar{\delta} \leq 1$, and $(\xi, \eta)$ is located above $k_{1}$, the solution is the rarefaction wave $R_{1}$.

(iii) As $(\xi, \eta) \in \mathcal{D}$, the solution is

$$
\left\{\begin{array}{l}
\rho(\xi, \eta)=\frac{1}{\gamma}\left[\left(1+\frac{\gamma-1}{2 \sin \delta} \xi\right) \tan ^{2} \bar{\delta}\right]^{\frac{2}{\gamma-1}} \\
u(\xi, \eta)=\left(\xi-\frac{1}{\sin \delta}\right) \tan ^{2} \bar{\delta} \\
v(\xi, \eta)=\eta
\end{array}\right.
$$

(iv) $\mathrm{As}-\frac{2}{\gamma-1} \leq \xi \sin \bar{\delta}+\eta \cos \bar{\delta} \leq 1$, and $(\xi, \eta)$ is located below $k_{2}$, the solution is the rarefaction wave $R_{2}$.

(v) In the rest part of the $(\xi, \eta)$ plane, the solution is the vacuum state, i.e. $\rho(\xi, \eta)=0$.

\section{A.3 Zhang-Zheng's exact spiral solution}

We take an exact spiral solution, or Zhang-Zheng's spiral solution, from [61] with $\gamma=2$. See also the references therein. The initial data is taken as

$$
(\rho, u, v)(x, y, 0)=\left(\rho_{0}, \sqrt{2 p^{\prime}\left(\rho_{0}\right)} \sin \theta,-\sqrt{2 p^{\prime}\left(\rho_{0}\right)} \cos \theta\right),
$$

where $\rho_{0}>0$ is an arbitrary parameter. We use the polar coordinate $(x, y)=(R \cos \theta, R \sin \theta)$. The solution consists of an inner part and an outer part. The inner solution takes the form

$$
(\rho, u, v)(x, y, t)=\frac{1}{2 t}\left(\frac{R^{2}}{4 t}, x+y,-x+y\right)
$$

if $R \leq 2 t \sqrt{p^{\prime}\left(\rho_{0}\right)}$. The outer solution is written as, if $R>2 t \sqrt{p^{\prime}\left(\rho_{0}\right)}$,

$$
\begin{aligned}
& \rho=\rho_{0}, \\
& u=\left(2 t p^{\prime}\left(\rho_{0}\right) \cos \theta+\sqrt{2 p^{\prime}\left(\rho_{0}\right)} \sqrt{R^{2}-2 t^{2} p^{\prime}\left(\rho_{0}\right)} \sin \theta\right) / R, \\
& v=\left(2 t p^{\prime}\left(\rho_{0}\right) \sin \theta-\sqrt{2 p^{\prime}\left(\rho_{0}\right)} \sqrt{R^{2}-2 t^{2} p^{\prime}\left(\rho_{0}\right)} \cos \theta\right) / R .
\end{aligned}
$$

This spiral solution has finite energy and vorticity in any bounded domain. The number of revolutions of the spiral approaches to infinity as we move to the center. 


\section{A.4 The 2-D four-wave Riemann problem}

It is well known that for conservation laws in one spatial dimension the interaction of elementary waves and the Riemann problem play the role of building blocks in the construction of solutions to more general initial value problems. The situation in two spatial dimensions is so totally different and notoriously difficult that very few analytic results are available. To understand substantial configurations and make problems accessible to study, in [60] 2-D four-wave Riemann problems were proposed. The flow configurations can be classified simply from the choice of initial data, which takes a constant state in each region,

$$
(\rho, u, v, p)(x, y, 0)=\left(\rho_{i}, u_{i}, v_{i}, p_{i}\right), \quad(x, y) \text { in the } i^{\text {th }} \text { quadrant, } \quad \mathrm{i}=1,2,3,4 .
$$

This means, for example, that initially the flow lies in a constant state $\left(\rho_{1}, u_{1}, v_{1}, p_{1}\right)$ in the first quadrant. Such a choice implies that only planar waves emit from a semi-axis to connect two neighboring states initially. We further put the following restriction in order to make the flow configurations as simple as possible but capture almost all essential 2-D phenomena:

Assumption. There is one and only one (1-D) planar elementary wave (a rarefaction wave, a contact discontinuity or a shock wave) emitting from each interface (a semi $x$-axis or a semi y-axis) that separates two distinct constant states.

For example, if the states $\left(\rho_{1}, u_{1}, v_{1}, p_{1}\right)$ and $\left(\rho_{2}, u_{2}, v_{2}, p_{2}\right)$ satisfy (A.5) with $(\mu, \nu)=(1,0)$, the wave emitting from the semi-axis $\{x=0, y>0\}$ is a single rarefaction wave $R_{12}^{+}$(resp. $R_{12}^{-}$) if $\rho_{1}>\rho_{2}$ (resp. $\rho_{1}<\rho_{2}$ ). With such an assumption, there are four planar elementary waves emitting from the half coordinate axes initially. We call (2.1) and (A.18) the 2-D four-wave Riemann problem conventionally or Zhang-Zheng's Riemann problem. Then the flow configurations are classified with the combination of these four waves. As shown in [60] and added in $[43,46]$ later on, there are 19 substantial cases. See also [37,61]. We will simulate each case in Section 4. These cases are enumerated below.

$$
\begin{aligned}
& 4 \mathrm{~S}: \quad S_{12}^{+} S_{23}^{+} S_{34}^{-} S_{41}^{-} ; \quad S_{12}^{+} S_{23}^{-} S_{34}^{+} S_{41}^{-} \\
& \text {4J: } \quad J_{12}^{-} J_{23}^{-} J_{34}^{-} J_{41}^{-} ; \quad J_{12}^{-} J_{23}^{+} J_{34}^{-} J_{41}^{+} \\
& 2 \mathrm{~J}+2 \mathrm{~S}: \quad S_{12}^{+} J_{23}^{+} J_{34}^{-} S_{41}^{-} ; \quad S_{12}^{-} J_{23}^{-} J_{34}^{+} S_{41}^{+} ; \quad J_{12}^{-} S_{23}^{-} J_{34}^{-} S_{41}^{+} ; \quad J_{12}^{+} S_{23}^{-} J_{34}^{-} S_{41}^{+} \\
& \text {4R: } \quad R_{12}^{+} R_{23}^{+} R_{34}^{-} R_{41}^{-} ; \quad R_{12}^{+} R_{23}^{-} R_{34}^{+} R_{41}^{-} \\
& 2 \mathrm{~J}+2 \mathrm{R}: \quad R_{12}^{+} J_{23}^{+} J_{34}^{-} R_{41}^{-} ; \quad R_{12}^{-} J_{23}^{+} J_{34}^{-} R_{41}^{+} ; \quad J_{12}^{+} R_{23}^{+} J_{34}^{+} R_{41}^{-} ; \quad J_{12}^{+} R_{23}^{+} J_{34}^{-} R_{41}^{-} \\
& 2 \mathrm{~J}+\mathrm{R}+\mathrm{S}: \quad R_{12}^{+} J_{23}^{+} J_{34}^{+} S_{41}^{+} ; \quad R_{12}^{-} J_{23}^{+} J_{34}^{+} S_{41}^{-} ; \quad J_{12}^{-} S_{23}^{-} J_{34}^{-} R_{41}^{-} ; \quad J_{12}^{+} S_{23}^{-} J_{34}^{+} R_{41}^{-} ; \quad J_{12}^{+} S_{23}^{-} J_{34}^{-} R_{41}^{-}
\end{aligned}
$$

\section{Acknowledgement}

Jiequan Li is partially supported by the Key Program from Beijing Educational Commission (KZ200910028002), 973 project (2006CB805902), PHR(IHLB) and NSFC (10971142). Huazhong Tang was partially supported by the National Basic Research Program under the Grant 2005CB321703, the National Natural Science Foundation of China (No. 10925101, 10828101), the Program for New 
Century Excellent Talents in University (NCET-07-0022), and the Doctoral Program of Education Ministry of China (No. 20070001036).

\section{References}

[1] M. Ben-Artzi and J. Falcovitz, A second-order Godunov-type scheme for compressible fluid dynamics, J. Comput. Phys., 55 (1984), 1-32.

[2] M. Ben-Artzi and J. Falcovitz, An upwind second-order scheme for compressible duct flows, SIAM J. Sci. Stat. Comput., 7 (1986), 744-768.

[3] M. Ben-Artzi, The generalized Riemann problem for reactive flows, J. Comput. Phys., 81(1989), 70-101.

[4] M. Ben-Artzi and J. Falcovitz, Generalized Riemann Problems in Computational Fluid Dynamics, Cambridge University Press, 2003.

[5] M. Ben-Artzi and J. Li, Hyperbolic balance laws: Riemann invariants and the generalized Riemann problem, Numer. Math. 106 (2007), 369-425.

[6] M. Ben-Artzi, J. Li and G. Warnecke, A direct Eulerian GRP scheme for compressible fluid flows, J. Comput. Phys., 218 (2006), 19-34.

[7] A. Birman, N. Y. Har'el, J. Falcovitz, M. Ben-Artzi, and U. Feldman, Operator-split computation of 3-D symmetric flow, CFD Journal, 10(2001), 37-43.

[8] G. Ben-Dor, Shock Wave Reflection Phenomena, Springer-Verlag, 1992.

[9] J. U. Brackbill, An adaptive grid with directional control, J. Comput. Phys., 108 (1993), 38-50.

[10] J. U. Brackbill and J. S. Saltzman, Adaptive zoning for singular problems in two dimensions, J. Comput. Phys., 46 (1982), 342-368.

[11] W. Cao, W. Huang, and R. D. Russell, A study of monitor functions for two-dimensional adaptive mesh generation, SIAM J. Sci. Comput., 20 (1999), 1978-1999.

[12] W. Cao, W. Huang and R. D. Russell, An $r$-adaptive finite element method based upon moving mesh PDEs, J. Comput. Phys., 149 (1999), 221-244.

[13] T. Chang and G.Q. Chen, Diffraction of planar shock along compressive corner, Acta. Math. Sci., 6 (1986), 241-257.

[14] T. Chang and G. Q. Chen, Some fundamental concepts about system of two spatial dimensional conservation laws, Acta Math. Sci. (English Ed.), 6 (1986), 463-474.

[15] T. Chang, G.Q. Chen, and S. Yang, On the 2-D Riemann problem for the compressible Euler equations I: Interaction of shocks and rarefaction waves, Dis. Cont. Dyn. Sys., 1(1995), 555-584. II: Interaction of contact discontinuities, Discrete Contin. Dyn. Syst., 6 (2000), 419-430.

[16] G.-Q. Chen and M. Feldman, Global solutions of shock reflection by large-angle wedges for potential flow, Annals of Math (2), to appear, 2007.

[17] S.X. Chen, Mach configuration in pseudo-stationary compressible flow, J. Amer. Math. Soc., 21 (2008), 63-100.

[18] A. van Dam and P.A. Zegeling, A robust moving mesh finite volume method applied to 1-D hyperbolic conservation laws from magnetohydrodynamics, J. Comput. Phys., 216 (2006), 526-546.

[19] A. van Dam and P.A. Zegeling, Balanced monitoring of flow phenomena in moving mesh methods, Commun. Comput. Phys., 7 (2010), 138-170.

[20] S.F. Davis and J.E. Flaherty, An adaptive finite element method for initial-boundary value problems for partial differential equations, SIAM J. Sci. Stat. Comput., 3 (1982), 6-27.

[21] Y. Di, R. Li, T. Tang, and P.W. Zhang, Moving mesh finite element methods for the incompressible Navier-Stokes equations, SIAM J. Sci. Comput., 26 (2005), 1036-1056.

[22] A. S. Dvinsky, Adaptive grid generation from harmonic maps on Riemannian manifolds, J. Comput. Phys., 95 (1991), 450-476.

[23] J. Falcovitz, G. Alfandary, and G. Hanoch. A 2-D conservation laws scheme for compressible ows with moving boundaries, J. Comput. Phys., 138 (1997), 83-102.

[24] J. Falcovitz and A. Birman, A singularities tracking conservation laws scheme for compressible duct flows, J. Comput. Phys., 115 (1994), 431-439. 
[25] E. Han, J.Q. Li and H.Z. Tang, An adaptive GRP scheme for compressible fluid flows, J. Comput. Phys. , 229 (2010), 1448-1466.

[26] W. Huang, Variational mesh adaptation: isotropy and equidistribution, J. Comput. Phys., 174 (2001), 903-924.

[27] J. Glimm, X. Ji, J. Li, X. Li, T. Zhang, P. Zhang and Y. Zheng, Transonic shock formation in a rarefaction Riemann problem for the 2-D compressible Euler equations, SIAM J. Appl. Math., 69 (2008), 720-742.

[28] E. Godlewski and P.-A. Raviart, Numerical Approximation of Hyperbolic Systems of Conservation Laws, Springer, 1996.

[29] S. K. Godunov, A finite difference method for the numerical computation and disontinuous solutions of the equations of fluid dynamics, Mat. Sb., 47 (1959), 271-295.

[30] J.Q. Han and H.Z. Tang, An adaptive moving mesh method for multidimensional ideal magnetohydrodynamics, J. Comput. Phys., 220 (2007), 791-812.

[31] K. G. Guderly, The Theory of Transonic Flow, Pergamon Press, London, 1962.

[32] A. Kurganov and E. Tadmor, Solution of two-dimensional Riemann problems for gas dynamics without Riemann problem solvers, Numer. Meth. Part. Diff. Eqs., 18 (2002), 584-608.

[33] P. Lax, Computational fluid dynamics, J. Sci. Comput., 31 (2007), 185-193.

[34] J. Li, On the two-dimensional gas expansion for compressible Euler equations,SIAM J. Appl. Math, 62 (2001), 831-852.

[35] J. Li and G. Chen, The generalized Riemann problem method for the shallow water equations with bottom topography, Int. J. Numer. Methods Eng., 65 (2006), 834-862.

[36] J. Li, T. Liu and Z. Sun, Implementation of the GRP scheme for computing radially symmetric compressible fluid flows, J. Comput. Phys., 228 (2009) 5867-5887.

[37] J. Li, T. Zhang and S. Yang, The Two-dimensional Riemann Problem in Gas Dynamics, Addison Wesley Longman, 1998.

[38] J. Li and Y. Zheng, Interaction of rarefaction waves of the two-dimensional self-similar Euler equations, Arch. Rat. Mech. Anal., 193 (2009), 623-657.

[39] J. Li and Y. Zheng, Interaction of Four Rarefaction Waves in the Bi-Symmetric Class of the TwoDimensional Euler Equations, Comm. Math. Phys., 296(2010), 303-326.

[40] J. V. Neumann, Oblique reflection of shocks, Navy Department, Bureau of Ordance, Explosive Research Report, No.12, 1943.

[41] R. Li, T. Tang, and P.W. Zhang, Moving mesh methods in multiple dimensions based on harmonic maps, J. Comput. Phys., 170 (2001), 562-588.

[42] R. Li, T. Tang, and P.W. Zhang, A moving mesh finite element algorithm for singular problems in two and three space dimensions, J. Comput. Phys., 177 (2002), 365-393.

[43] X.D. Liu and P.D. Lax, Solution of two-dimensional Riemann problems of gas dynamics by positive schemes, SIAM J. Sci. Comput., 19 (1998), 319-340.

[44] K. Miller and R. N. Miller, Moving finite element. I, SIAM J. Numer Anal., 18 (1981), 1019-1032.

[45] A. Rault, G. Chiavassa and R. Donat, Shock-vortex interactions at high Mach numbers, J. Sci. Comput., 19 (2003), 347-371.

[46] C.W. Schulz-Rinne, J.P. Collins, and H.M. Glaz, Numerical solution of the Riemann problem for twodimensional gas dynamics, SIAM J. Sci. Comput., 14 (1993), 1394-1414.

[47] W.C. Sheng and G. Yin, Transonic shock and supersonic shock in the regular reflection of a planar shock, Z. Angew. Math. Phys., 60 (2009), 438-449.

[48] G.A. Sod, A survey of several finite difference methods for systems of nonlinear hyperbolic conservation laws, J. Comput. Phys., 27 (1978), 1-31.

[49] H.Z. Tang, A moving mesh method for the Euler flow calculations using a directional monitor function, Commun. Comput. Phys., 1 (2006), 656-676

[50] H.Z. Tang and T. Tang, Adaptive mesh methods for one- and two- dimensional hyperbolic conservation laws, SIAM J. Numer. Anal., 41 (2003), 487-515.

[51] T. Tang, Moving mesh methods for computational fluid dynamics, Contem. Math., 383 (2005), $185-218$.

[52] E.F. Toro, Riemann Solvers and Numerical Methods for Fluid Dynamics: A Practical Introduction, Springer, 1997. 
[53] B. van Leer, Towards the ultimate conservative difference scheme V, J. Comput. Phys., 32 (1979), 101-136.

[54] C.W. Wang, H.Z. Tang and T.G. Liu, An adaptive ghost fluid finite volume method for compressible gas-water simulations, J. Comput. Phys., 227 (2008), 6385-6409.

[55] D. Wang and X. Wang, A three-dimensional adaptive method based on the iterative grid redistribution, J. Comput. Phys., 199 (2004), 423-436.

[56] A. Winslow, Numerical solution of the quasi-linear Poisson equation, J. Comput. Phys., 1 (1967), 149-172.

[57] P. Woodward and P. Colella, The numerical simulation of two-dimensional fluid flow with strong shocks, J. Comput. Phys., 54 (1984), 115-173.

[58] S.J. Wu, Mathematical analysis of vortex sheets, Comm. Pure Appl. Math., 59 (2006), 1065-1206.

[59] P.A. Zegeling, W.D. de Boer, and H.Z. Tang, Robust and efficient adaptive moving mesh solution of 2-D Euler equation, Contem. Math., 383 (2005), 375-386.

[60] T. Zhang and Y. Zheng, Conjecture on the structure of solutions of the Riemann problem for twodimensional gas dynamics systems, SIAM J. Math. Anal., 21 (1990), 593-630.

[61] Y. Zheng, Systems of Conservation Laws: Two-dimensional Riemann Problems, Birkhäuser, 2001. 